\title{
XI. Ueber die Mineralfundstätte von Branchville, Connecticut.
}

\author{
4. Abhandlung. \\ Der Spodumen und seine Zersetzungsproducte. \\ Von \\ G. J. Brush und Edw. S. Dana in New-Haven $\left.{ }^{*}\right)$. \\ (Hierzu Tafel IV und 6 Holzschnitte.)
}

In dieser Abhandlung sollen die Resultate der Untersuchung des Spodumen von Branchville, Connecticut, und der mannigfaltigen Mineralien, welche aus seiner Umwandlung hervorgehen, mitgetheilt werden $\left.{ }^{* *}\right)$. Der-

*) Aus dem Amer. Journ. of Sc. 20, 357 (Oct. 1880) von den Verf. mitgetheilt. Die Herren Brush und Dan a hatten ausserdem die Freundlichkeit, die ganze Auflage der zugehörigen Tafel IV nebst den Originalholzschnitten der Red. zur Verfügung zu stellen, wofür Denselben auch an dieser Stelle der schuldige Dank ausgesprochen sei. Die Red.

**) Eine umfangreiche und wichtige Arbeit über "Spodumen und seine Zersetzungsproducte aus den Granitgängen von Hampshire Co., Mass., wurde vor Kurzem von Herrn A. A. Julien in den "Annals of the New-York Acad. of Sc. 1, Nr. X." veroffentlicht. Wir müssen bemerken, dass die meisten unserer Resultate, mit Einschluss sámmtlicher Analysen, vollendet waren vor dem Erscheinen derjenigen des Herrn Julie n und ehe wir von den letzteren weitere Kenntniss hatten, als sie aus der vorläufigen Mittheilung dieses Autors über Gymatolith (s. diese Zeitschr. 4, 86) zu entnehinen war. Indessen war es uns bekannt, dass Herr Julien mit dieser Untersuchung beschäftigt sei und bereits seit einigen Jahren daran arbeite, und hielten wir es desshalb für passend, unsere Publication erst erfolgen zu lassen, nachdem jene erschienen war. Wie man sehen wird, bestätigen unsere Resultate, obgleich unabhängig erzielt und auf Material eines andern Fundortes begründet, in vielen Fällen die des Herrn Julic $n$, und dieser Umstand scheint uns geeignet, das Interesse an dem Gegenstande noch zu vermehren. Unsere Schlussfolgerungen sind indess in manchen Beziehungen abweichende: wir haben gefunden, dass Cymatolith kein eigentliches Mineral, sondern nur ein mechanisches Gemenge von Albit und Muscovit ist. Diese Thatsache, zusammen mit der Existenz der analogen zusammengesetzten Substanz, dem $\beta$-Spodumen, ermöglicht es jetzl, eine verständliche und vollständige Erklärung aller der Umwandlungen, welche in diesem sehr interessanten Falle von Pseudomorphismus vor sich gegangen sind, zu geben. 
selbe ist nach dem Feldspath, Glimmer und Quarz das wichtigste unter den ursprünglichen Mineralien der Fundstätte und kommt, wenn auch meist in verändertem Zustande, in sehr grosser Menge vor.

\section{A. Unzersetzter Spodumen.}

Der grösste Theil des unveränderten Spodumens tritt in verworrenen krystallinischen Massen mit deutlicher Spaltbarkeit auf, aber selten mit einer Andeutung ausgebildeter Krystallformen. Man kann das Mineral fast rein, nur mit etwas Albit gemengt; in Blöcken von mehreren hundert Pfund erhalten. Dieser derbe Spodumen ist trübe weiss, blos stellenweise bunt gefärbt und nur zum Theil durchscheinend; die Spaltungsflächen sind oft von zarten Dendriten von Manganoxyd bedeckt. Die begleitenden Mineralien sind, ausser dem Albit und ein wenig Quarz und Glimmer: Apatit, Lithiophilit, Columbit, Granat, Uraninit und verschiedene andere durch Zersetzung entstandene Uranmineralien.

Abgesehen von dieser derben Varietät kommt der Spodumen auch in unverändertem Zustande als Kern deutlicher pseudomorpher Krystalle vor. Diese letzteren besitzen oft eine enorme Grösse und sind meistens in derben Quarz eingewachsen, ragen aber auch zuweilen in den Albit hinein. Der Kern von Spodumen (s. unten und Fig. 1a, 5, 8, 14, Taf. IV) ist in jedem Falle scharf von dem umgebenden zersetzten Mineral getrennt; ihr Ansehen beweist, dass die Krystalle ursprünglich von seltener Schönheit gewesen sein müssen. Einer der besten, die wir fanden, hat, soweit er in Quarz eingewachsen ist, eine Länge von drei Fuss, bei acht Zoll Breite und zwei Zoll Dícke; der unveränderte Spodumen, von schöner Amethystfarbe, bildet ungefähr ein Viertel des Ganzen und erstreckt sich ziemlich regelmässig durch die Mitte des Krystalls, ist aber leider von zahlreichen Rissen und Sprügen durchzogen, so dass er seine fruhere Durchsichtigkeit, meist eingebussst bat; die äusseren Partien des Krystalls bestehen hauptsächlich aus $\beta$-Spodumen mit geringen Mengen von Cymatolith und Albit. Ein anderer in Quarz eingewachsener Krystall wurde auf eine Länge von uber vier Fuss blosgelegt. Es ist nicht möglich, solche Krystalle ganz zu gewinnen, es wurden aber Bruchstücke frei gemacht, welche quer gegen das Prisma eine Breite von über ein Fuss und eine Dicke von zwei bis vier Zoll besassen. Im Habitus gleichen die Krystalle meist denen von Norwich, Massachusetts, d. h. sind im Allgemeinen durch die Entwickelung des Orthopinakoids breit tafelförmig und verhältnissmässig dưnn; nicht selten sind auch die Endflächen gut ausgebildet. Gelegentlich wurden auch dicke Krystalle von rechtwinkelig prismatischer Form, mehr dem eigentlichen Pyroxen ähnlich, beobachtet. 
In den besseren Stucken ist der Spodumen vollkommen durchsichtig, theils farblos, theils schön rosa oder amethystroth gefärbt. Er zeigt die prismatische Spaltbarkeit mit ungewöhnlicher Vollkommenheit, die nach dem Klinopinakoid unregelmässig. Der Winkel der ersteren, 870 13', konnte sehr genau gemessen werden.

Ghemische Zusammensetzung. Eine Analyse des durchsichtigen rothen Spodumen wurde von Herrn S. L. Penfield mit folgenden Resultaten ausgefuhrt: Spec. Gew. 3,193.

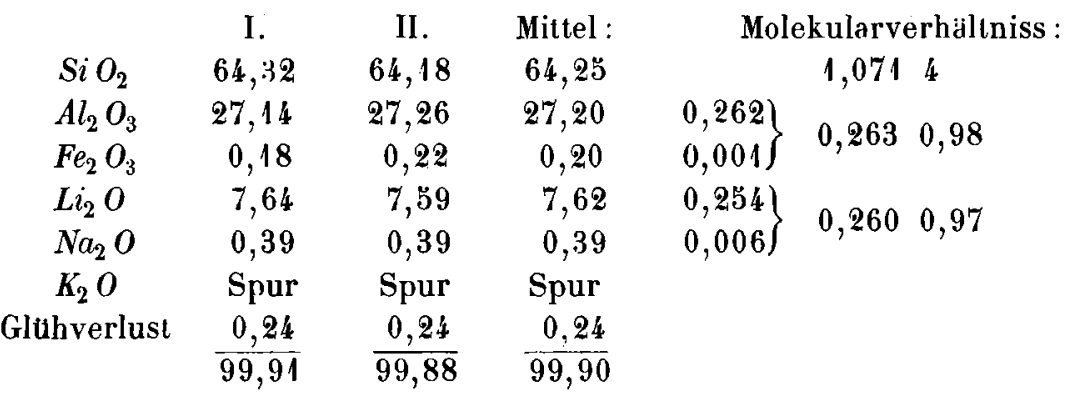

Das Verbältniss von $\mathrm{Li}_{2} \mathrm{O}: \mathrm{Al}_{2} \mathrm{O}_{3}: \mathrm{SiO}_{2}$ ist $=1: 1: 4$; dem entspricht das Sauerstoffverhältniss $1: 3: 8$, wie es bereits $1850 \mathrm{~B} \mathrm{rush} \mathrm{(Am.} \mathrm{Journ.}$ (2) 10,370) aus Analysen des Minerals von Massachusetts erhielt. Die Formel ist also, unter Vernachlässigung der sehr kleinen Menge Natron:

$$
\mathrm{Li}_{2} \mathrm{Al}_{2} \mathrm{Si}_{4} \mathrm{O}_{12}
$$

Dieses Resultat stimmt vollkommen itberein mit dem von Dölter (s. diese Zeitschr. 4, 93) bei seiner Untersuchung uber die Zusammensetzung des Spodumen erhaltenen und demjenigen von Juli en (1. c. S. 325). Nur der Procentgehalt des Lithions ist in der Penfie ld'schen Analyse höher und der des Natrons geringer, als in irgend einer der fruher publicirten. So fand z. B. Döl ter in dem Spodumen von Norwich 7,04 $\mathrm{Li}_{2} \mathrm{O}, 1,10 \mathrm{Na}_{2} \mathrm{O}$ und 0,12 $\mathrm{K}_{2} \mathrm{O}$, in dem von Brasilien 7,09 $\mathrm{Li}_{2} \mathrm{O}$ und $0,98 \mathrm{Na}_{2} \mathrm{O}$; Julien erhielt bei dem Mineral von Goshen 6,89 $\mathrm{Li}_{2} \mathrm{O}, 0,99 \mathrm{Na}_{2} \mathrm{O}, 1,45 \mathrm{~K}_{2} \mathrm{O}$ und in dem von Chesterfield 6,99 $\mathrm{Li}_{2} \mathrm{O}, 0,50 \mathrm{Na}_{2} \mathrm{O}$ und $1,33 \mathrm{~K}_{2} \mathrm{O}$. DöI ter giebt an, dass der von ihm für den Spodumen von Norwich gefundene Lithiongehalt eher zu klein als zu gross sei, und schreibt die Gegenwart des Natrons einer beginnenden Zersetzung zu. Die Richtigkeit dieser Ansicht scheint durch die obigen Analysen des Minerals von Branchville, welches gewiss in Bezug auf Reinheit und Frische Nichts zu wünschen übrig lässt, bestätigt zu werden. Die grosse Leichtigkeit, mit welcher der Spodumen durch Aufnahme von Kali und Natron, unter Verlust des Lithion, eine Veränderung erleidet, wird aus dem Folgenden ersichtlich werden. 


\section{B. Zersetzung des Spodumens.}

Als Producte der Umwandlung des Spodumens haben wir zwei Substanzen gefunden, welche beim ersten Anblick homogen zu sein scheinen, deren jede eine bestimmte chemische Zusammensetzung besitzt, und welche nichsdestoweniger nur innige mechanische Gemenge zweier Mineralien sind. Eine davon, von uns $\beta$-Spodumen genannt, besteht aus Albit und einer neuen Lithionverbindung, welcher wir den Namen Eukryptit gegeben baben; die andere, der Cymatolith, ist ein Aggregat von Albit und Muscovit. Unabhängig davon fanden wir Albit, Mikroklin, Muscovit und Killinit. Die beiden zusammengesetzten Substanzen, sowie die letztgenannten Mineralien, mit Ausnahme des Glimmers, kommen in deutlichen Pseudomorphosen von der Form des Spodumens vor. Der Glimmer, soweit er nicht der constanten Association mit dem Albit angehört, spielt nur eine untergeordnete Rolle. Endlich kommen noch andere Pseudomorphosen von zusammengesetztem Charakter vor, welche, wie Herr Julien es richtig ausdruckt, aus Ganggranit bestehen.

Im Folgenden sollen zuerst die physikalischen und chemischen Eigenschaften der verschiedenen Mineralien (mit Einschluss der beiden Aggregate) einzeln angegeben und alsdann die Art, wie dieselben mit einander associirt sind, eingehender beschrieben werden.

\section{Die Zersetzungsproducte.}

1. $\beta$-Spodumen.

Die Substanz, welcher wir der Bequemlichkeit wegen, da wir nicht glauben, dass sie einen eigenen Namen verdient, den obigen beigelegt haben, scheint die erste Stufe in der Zersetzung des Spodumens zu bezeichnen.

Physikalische Eigenschaften. Das Mineral ist derb, anscheinend homogen und besitzt eine ziemlich undeutliche faserige bis stängelige Structur, stets senkrecht gegen die benachbarte Oberfläche des ursprunglichen Krystalls. Härte 51/2-6, spec. Gew. 2,644-2,649. Farbe milchweiss bis schwach grüulichweiss. Durchscheinend. Schmelzbarkeit $21 / 4$.

Ghemische Zusammensetzung. Es wurden von drei verschiedenen Stucken Analysen durch Herrn S. L. Penfield ausgeführt. Das Material der ersten war dem oben beschriebenen Krystall entnommen, dessen Inneres aus dem durchsichtigen rothen Spodumen bestand, und bildete den äusseren Theil desselben (vergl. Fig: 5). Die Trennungslinie beider war vollkommen scharf, so dass die Reinheit des analysirten Materials ausser Zweifel steht. Die Resultate der Analyse sind die folgenden: 
Nr. 1 (spec. Gew. 2,649).

I. II. Mittel: Molekularverhältniss:

\begin{tabular}{|c|c|c|c|c|c|}
\hline $\mathrm{SiO}_{2}$ & 61,35 & 61,42 & 61,38 & & 1,0234 \\
\hline $\mathrm{Al}_{2} \mathrm{O}_{3}$ & 26,26 & 25,74 & 26,00 & $0,253\}$ & \\
\hline $\mathrm{Fe}_{2} \mathrm{O}_{3}$ & 0,24 & 0,24 & 0,24 & $0,002\}$ & $0,255 \quad 0,99$ \\
\hline $\mathrm{Li}_{2} \mathrm{O}$ & 3,63 & 3,59 & 3,61 & $0,120\}$ & 0954099 \\
\hline $\mathrm{Na}_{2} \mathrm{O}$ & 8,32 & 8,25 & 8,29 & $0,134\}$ & \\
\hline $\mathrm{K}_{2} \mathrm{O}$ & Spur & Spur & Spur & & \\
\hline
\end{tabular}

Gluhverlust $\frac{0,46}{100,26} \quad \frac{0,46}{99,70} \quad \frac{0,46}{99,98}$

Die zweite, zur Analyse verwendete Portion stammte von einem Fragment eines grossen und ganz umgewandelten Krystalls (Dimensionen $9: 8: 2^{1} / 2$ Zoll), welcher meist aus Cymatolith bestand, in den der $\beta$-Spodumen allmälig uberzugehen schien. Ein einziges Bruchstuck, quer gegen das Prisma, war aus beiden Mineralien derart zusammengesetzt, dass die faserige Structur des einen sich in dem andern fortsetzte (wie es Figur $1 \mathrm{~b}$ zeigt). Die Analyse lieferte:

Nr. 2 (spec. Gew. 2,644).

I. II. Mittel : Molekularverhältniss:

\begin{tabular}{|c|c|c|c|c|c|}
\hline \multirow{2}{*}{$\begin{array}{l}\text { SiC } \\
\mathrm{Al}_{2}\end{array}$} & 61,46 & 61,57 & 61,51 & \multicolumn{2}{|r|}{1,0254} \\
\hline & ht besti & 26,56 & 26,56 & & $0,258 \quad 1$ \\
\hline $\mathrm{Li}_{2} \mathrm{O}$ & 3,55 & 3,44 & 3,50 & $0,117)$ & \\
\hline $\mathrm{Na}_{2} \mathrm{O}$ & 8,15 & 8,13 & 8,14 & $0,131\}$ & $0,249 \quad 0,97$ \\
\hline$K_{2} O$ & 0,15 & 0,15 & 0,15 & $0,001)$ & \\
\hline
\end{tabular}

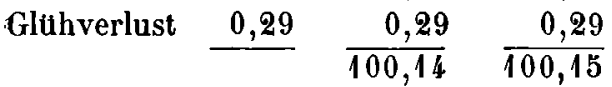

Die dritte Portion endlich bildete einen Theil eines kleineren und gut ausgebildeten Krystalls von vollständiger prismatischer Gestalt. Derselbe bestand im Inneren aus Spodumen, während der $\beta$-Spodumen den grösseren Theil des Ganzen ausmachte, und batte endlich eine dunne Rinde von Cymatolith. Die analysirte Partie war, soweit man es mit dem Auge beurtheilen konnte, vollkommen rein und homogen, deutlich durchscheinend und von grünlichweisser Farbe. Die Zerlegung ergab:

Nr. 3 (spec. Gew. 2,649).

I.

$\begin{array}{ll}\mathrm{SiO}_{2} & 61,78 \\ \mathrm{Al}_{2} \mathrm{O}_{3} & 26,57 \\ \mathrm{Li}_{2} \mathrm{O} & \\ \mathrm{Na}_{2} \mathrm{O} & \\ \mathrm{K}_{2} \mathrm{O} & \end{array}$

Glühverlust
II. Mittel :

$61,64 \quad 61,71$

$26,69 \quad 26,63$

3,83

8,16

Spur

$\frac{0,21}{100,53} \quad \frac{0,21}{100,54}$

8,16

Spur
Molekularverhältniss :

\begin{tabular}{|c|c|}
\hline & 1,028 \\
\hline & 0,259 \\
\hline $\left.\begin{array}{l}0,128 \\
0,132\end{array}\right\}$ & 0,260 \\
\hline
\end{tabular}


Bei der Vergleichung der Mittelwerthe dieser drei Gruppen von AnaJysen ersieht man sogleich, dass dieselben sebr nahe mit einander ubereinstimmen, in der That so nahe, wie man es nur erwarten könnte von drei, nach einander mit einem und demselben Material ausgeführten Analysen. Trotzdem waren die drei Portionen, wie aus dem Vorhergehenden ersichtlich, ganz unabhängig von einander, von verschiedenen Schichten entnommen und abweichend in der Art ihrer Association; die Uebereinstimmung unter den Resultaten wird dadurch um so auffallender. Das aus allen Analysen folgende Molekularverhältniss ist

$$
\stackrel{\mathrm{I}}{\mathrm{R}_{2}} \mathrm{O}: \mathrm{R}_{2} \mathrm{O}_{3}: \mathrm{SiO}_{2}=1: 1: 4
$$

d. i. dasjenige des Spodumen selbst; von welchem sich die Substanz nur dadurch unterscheidet, dass in derselben die Hälfte des Lithium durch die äquivalente Menge Natrium ersetzt ist. Die Formel ist also:

$$
\begin{aligned}
(\mathrm{Li}, \mathrm{Na})_{2} \mathrm{Al}_{2} \mathrm{Si}_{4} \mathrm{O}_{12} & =\mathrm{Li}_{2} \mathrm{Al}_{2} \mathrm{Si}_{4} \mathrm{O}_{12}+\mathrm{Na}_{2} \mathrm{Al}_{2} \mathrm{Si}_{4} \mathrm{O}_{12} \\
\text { oder } & =\mathrm{Li}_{2} \mathrm{Al}_{2} \mathrm{Si}_{2} \mathrm{O}_{8}+\mathrm{Na}_{2} \mathrm{Al}_{2} \mathrm{Si}_{6} \mathrm{O}_{16}
\end{aligned}
$$

Es wird weiterhin gezeigt werden, dass die Formel (2) die richtige ist.

So weit scheinen die mitgetheilten Thatsachen genugend für den Beweis, dass das Mineral homogen sei und eine bestimmte Zusammensetzung besitze; trotzdem existiren noch andere, für diese Frage nicht minder zu berúcksichtigende Facta.

Zunächst wurde von Herrn Pen field gefunden, dass das Mineral, obgleich mit Säuren gelatinirend, nicht ganz zersetzt wird, sondern sich vielmehr bei der Behandlung mit Salzsäure in zwei Portionen theile, eine lösliche (A) und einen unlöslichen Rtickstand (B), von denen letzterer auch die von dem löslichen Antheil ausgeschiedene Kieselsäure enthält. Die Resultate dreier Zerlegungen waren :

A. (lösl. i. $\mathrm{HCl}$ ) B. (unlösl. i. $\mathrm{HCl}$,

\begin{tabular}{rcccc}
\multicolumn{5}{c}{ mit $\mathrm{SiO}_{2}$ von A.) } \\
$\mathrm{Nr} .1$ & $(17,97)$ & 82,03 & $=100$ \\
-2 & 16,65 & 83,01 & $=99,66$ \\
-3 & 17,91 & 82,18 & $=$ & 100,09
\end{tabular}

Von der zweiten Portion wurden vollständige Analysen sowohl des löslichen, als des unlöslichen Antheils gemacht, und zwar unabhängig von den oben angefuhrten Totalanalysen derselben Substanz. Die Methode der Zerlegung war im Wesentlichen die folgende: - Ein Gramm der Substanz wurde mit $\mathrm{HCl}$ zersetzt, zur Trockne verdampft, dann mit Salzsäure befeuchtet und zum zweiten Male zur Trockne verdampft. Nach abermaligem Befeuchten mit Salzsäure wurde der lösliche Antheil (A oben) abfiltrirt und Thonerde nebst Alkalien in demselben nach den gewöhnlichen Methoden bestimmt. Der unlösliche Antheil, welcher die aus A abgeschiedene Kiesel- 
säure einschliesst, wurde vorher gewogen und dann mit $\mathrm{Na}_{2} \mathrm{CO}_{3}$ und (bei Nr. 3) mit etwas $\mathrm{KOH}$ gekocht. Hierdurch ging die Kieselsäure von $\mathbf{A}$ in Lösung; ; ihre Menge wurde, indem der unlösliche Rückstand gewogen wurde, durch Differenz bestimmt. Schliesslich wurde der unlösliche Theil vollstandig analysirt nach den ublichen Methoden. Die Resultate dieser Zerlegung waren folgende :

Nr. 2.

B. Unlöslich in $\mathrm{HCl}$ - und $\mathrm{SiO}_{2}$ von A 83,01

Unlöslicher Ruckstand nach der Behandlung mit Soda $\frac{67,56}{15,45}$

A. Löslich in $\mathrm{HCl}(16,65)$, dazu $\mathrm{Si} \mathrm{O}^{2}$ mit Soda ausgezogen $\quad 32,10$

Die beiden Antheile, in welche das Mineral durch die Salzsäure zerlegt worden ist, sind somit:
A. Löslich
32,10
B. Unlöslich
$\frac{57,56}{99,66}$

Die Zusammensetzung von A wurde gefunden, wie folgt:

$\begin{array}{lccc} & \text { A. Nr. 2. } & \text { Auf } 100 \text { berechnet: } & \text { Ber. n. d. Formel: } \\ \mathrm{SiO}_{2} & 15,45 & 48,13 & 47,51 \\ \mathrm{Al}_{2} \mathrm{O}_{3} & 13,00 & 40,50 & 40,61 \\ \mathrm{Li}_{2} \mathrm{O} & 3,50 & 10,90 & 11,88 \\ \mathrm{~K}_{2} \mathrm{O} & 0,15 & 0,47 & - \\ & \overline{32,10} & \frac{0,40}{100,00} & \overline{100,00}\end{array}$

Das hieraus sich ergebende Molekularverhältniss ist $\mathrm{SiO}_{2}: \mathrm{Al}_{2} \mathrm{O}_{3}: \mathrm{Li}_{2} \mathrm{O}$ $=2: 1: 1$, entsprechend der Formel $\mathrm{Li}_{2} \mathrm{Al}_{2} \mathrm{Si}_{2} \mathrm{O}_{3}$, nach welcher die obigen, mit den Resultaten der Analyse gut ubereinstimmenden Zahlen berechnet sind.

Die Zusammensetzung des unlöslichen Theils ergab sich:

B. Nr. 2. Auf 100 berechnet: Ber. n. d. Formel:

$\begin{array}{lrrr}\mathrm{SiO}_{2} & 46,06 & 68,18 & 68,62 \\ \mathrm{Al}_{2} \mathrm{O}_{3} & 13,56 & 20,07 & 19,56 \\ \mathrm{Na}_{2} \mathrm{O} & 7,94 & 11,75 & 11,82 \\ & \overline{67,56} & \underline{100,00} & \frac{100,00}{}\end{array}$

Aus dieser Analyse folgt das Verhältniss $\mathrm{SiO}_{2}: \mathrm{Al}_{2} \mathrm{O}_{3}: \mathrm{Na}_{2} \mathrm{O}=6: 1,07: 1,00$, d. $\mathfrak{i}$. fast ganz genau $6: 1: 1$, dasjenige des Albit; es ist somit die Formel des unlöslichen Theiles: $\mathrm{Na}_{2} \mathrm{Al}_{2} \mathrm{Si}_{6} \mathrm{O}_{16}$.

In gleicher Weise wurde eine Untersuchung der Portion Nr. 3 vorgenommen, hier war aber die Trennung etwas weniger vollkommen gelungen, als in Nr. 2. Die erste Behandlung mit Säure hatte nämlich, wie sich durch die Anwesenheit von Lithium in B zeigte, eine ganz kleine Menge von dem löslichen Mineral zuruckgelassen, und bei der dann folgenden Behandlung 
des unlöslichen Theils, bei welcher auch $K O H$ angewendet wurde, scheint eine beginnende Zersetzung des Albit eingetreten zu sein. In Folge dessen sind die Resultate dieses Versuches schwerlich werth, notirt zu werden, genugten aber vollkommen, die an $\mathrm{Nr} .2$ erhaltenen zu bestätigen.

Das soeben beschriebene Verhalten den' chemischen Agentien gegenuber beweist also, dass die provisorisch mit dem Namen $\beta$-Spodumen belegte Substanz kein eigentliches Mineral, sondern nur ein sehr gleichförmiges Gemenge zweier Substanzen ist; die eine derselben, von uns Eukryptit. genannt, löst sich unter Gelatiniren in Salzsäure und hat die Zusammensetzung $\mathrm{Li}_{2} \mathrm{Al}_{2} \mathrm{Si}_{2} \mathrm{O}_{8}$, die andere, durch Säuren nicht angreifbare, ist Albit $\mathrm{Na}_{2} \mathrm{Al}_{2} \mathrm{Si}_{6} \mathrm{O}_{16}$. Der wahre Ausdruck der chemischen Zusammensetzung des $\beta$-Spodumen ist also der auf S. 196 unter (2) gegebene. Dass es sich wirklich um ein mechanisches Gemenge und nicht (wenn dies uberhaupt möglich wäre) um eine durch die Säure zerlegte Molekularverbindung handelt, wird dadurch erwiesen, dass der unlösliche Rückstand (B), welcher nach der Behandlung mit Natriumcarbonat übrig bleibt, bei der mikroskopischen Prufung eine krystallin ische Beschaffenheit und zwar die besondere halbfaserige Structur zeigte, welche den weiter unten beschriebenen pseudomorphen Albit charakterisirt.

Die aus den chemischen Beobachtungen gezogenen Schiusse uber die zusammengesetzte Natur des $\beta$-Spodumen werden ferner bestätigt durch die mikroskopische Untersuchung von Dünnschliffen, und letztere liefert zudem eine sehr befriedigende Bestimmung des Krystallsystems des neuen Lithiumminerals. Es wurde eine Reihe von Dunnschliffen hergestellt, theils parallel der faserigen Structur, d. i. rechtwinkelig zu den Flächen des ursprunglichen Spodumenkrystalls, theils quer gegen die Fasern und folglich parallel den ursprünglichen Prismenflächen. Die ersteren schienen beim ersten Anblick unter dem Mikroskop keinen Beweis fưr eine Inhomogenităt. der Substanz zu liefern; die anscheinend runden Fasern, obgleich im Allgemeinen parallel, doch mit ganz gewundenen Umrissen, sind so dicht aufeinander gepackt, dass es unmöglich ist, uber die Gegenwart oder Abwesenheit einer Substanz zwischen denselben Etwas auszusagen; das Ganze zeigt Aggregatpolarisation. Gelegentlich finden sich jedoch in der parallelfaserigen Masse Unregelmässigkeiten, deren Ansehen nicht unpassend mit dem der Faserung des Holzes in der Năhe eines Knotens, wie sich solche an einem polirten Brete zeigt, zu vergleichen wäre. An diesen Stellen waren die Fasern so gekrummt, von so unregelmässigen Umrissen und so weit von einander getrennt, dass es deutlich erkannt werden konnte, dass dieselben nur Einschlusse in einer umschliessenden Grundmasse bilden. An anderen Stellen bildete dieses umgebende Material einschlussfreie Partien, deren Structur sich im polarisirten Lichte ubereinstimmend mit derjenigen gewöhnlichen Albites ergab, und in welche die nadelförmigen Fasern des 
anderen Minerals hineinragten (s. Fig. 15*), $\alpha=$ Albit). Ein ähnlich befriedigendes Resultat wurde ferner an den Rändern der Dünnschliffe erzielt, wo zuweilen ein Grad der Dunne erreicht wurde, wie er im ganzen Schliff zu erhalten nicht möglich war: daselbst sah man alsdann die Fasern deutlich von einander und von dem umgebenden Albit getrennt. Sie sind im Allgemeinen gerade und parallel, aber nicht selten auch von mehr oder weniger unregelmässiger Gestalt; so sind namentlich verästelte Formen von korallen-

Fig. 15.

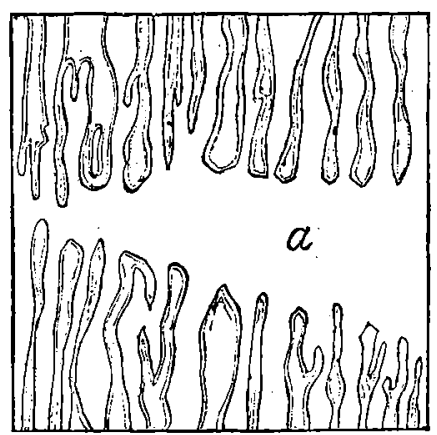

Fig. 16.

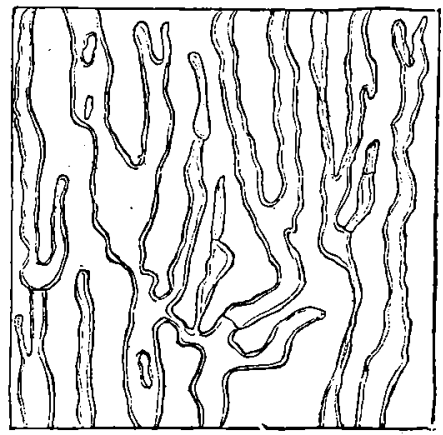

ähnlichem Bau nicht ungewöhnlich. Die letzteren Formen sind in Fig. 16 dargestellt, jedoch mit viel unregelmässigeren und gröberen Fasern, als es im Allgemeinen der Fall ist (vergl. auch Fig. 19). Anscheinend sind die Fasern rund, doch sind die Umrisse gewöhnlich unbestimmt, und man muss den Focus des Mikroskops wiederholt verändern, um ihre Form zu erkennen. Die Erklärung aller dieser Unregelmässigkeiten der Umrisse ergab sich jedoch, wie weiter unten ersichtlich, durch die Resultate, welche mit den Schliffen senkrecht zu den Fasern erhalten wurden. Indessen gaben die hier in Rede stehenden Schliffe noch Gelegenheit zu einigen anderen Beobachtungen: bei der Untersuchung zwischen gekreuzten Nicols zeigte sich die Auslöschung parallel der Längsrichtung der Fasern; diese letzteren hatten nicht selten Quersprünge, welche auf eine Spaltbarkeit hindeuteten. Die Form der Endausbildung der Nadeln, obgleich in den oben beschriebenen Fällen (Fig. 15) dieselbe ganz vorhanden zu sein schien, konnte nicht mit Sicherheit ermittelt werden. In vielen, wahrscheinlich den meisten Fällen liefen die Nadeln allmälig in eine feine Spitze aus, während sie in anderen durch eine flache Pyramide geschlossen zu sein schienen.

$\mathrm{Zu}$ weit befriedigenderen und sichereren Resultaten fuhrte die Untersuchung der zweiten Reihe von Schliffen, derjenigen senkrecht zu den Fasern. Ein solcher liefert beim Drehen desselben im polarisirten Lichte

*) Diese und die folgenden Figuren (15-19) entsprechen einer $300-$ fachen Vergrösserung. 
folgendes auffallende und hubsche Bild : der ganze Schliff erscheint in unregelmässige Flecken (Albit) getheill, welche beim Drehen hell und dunkel werden und dem Ganzen ein sonderbar geflecktes Aussehen ertheilen; in dieser Grundmasse nun erblickt man, dicht und gleichförmig vertheilt, kleine Querschnitte einer anderen Substanz, manchmal gekrummt, im Allgemeinen aber unter Winkeln von $60^{\circ}$ oder $120^{\circ}$ gegen einander geneigt; dieselben bleiben beim Drehen zwischen gekreuzten Nicols unverändert. Die Erscheinung ist am geeignetsten aus den-beistehenden Fig. 17 und 18

Fig. 17.

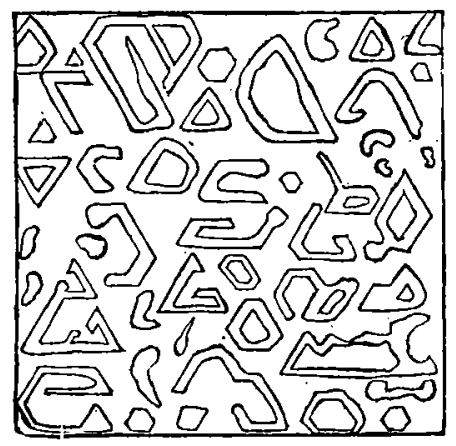

Fig. 18.

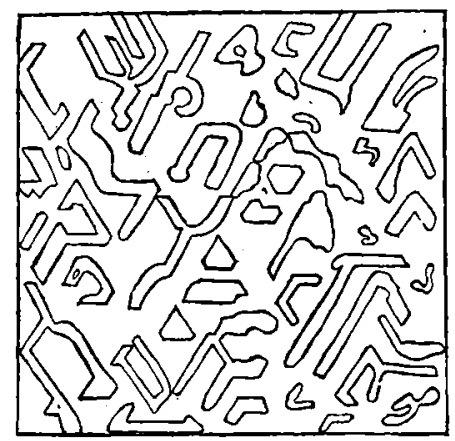

zu ersehen. Wenn eine starke Vergrösserung (z. B. 600 f.) angewendet und die Aufmerksamkeit zugleich auf eine kleine Partie beschränkt wird, so sieht man, dass diese schmalen Banden, welche einem oberflächlichen Blick bei schwacher Vergrösserung ganz unregelmässig erscheinen, im Gegentheil sich annäbernd in paralleler Stellung befinden. Die soliden Theile sind triangulär oder hexagonal im Umriss; die Banden beruhren einander unter Winkeln von $60^{\circ}$ oder $120^{\circ}$, manchmal so, dass sie vollständige Ringe bilden, und sind alle mehr oder weniger gerundet. Mit einem Worte, die Structur ist dieselbe, wie die des regelmässigsten Pegmatites oder "Schriftgranites ", und die Entstehung dieselbe, wie die des Quarzes im Feldspath bei letzterem, nämlich veranlasst durch die gehinderte Krystallisation des neuen in Rede stehenden Minerals im Albit. Die Formen, in Uebereinstimmung mit dem optischen Befund parallel und senkrecht zu den Fasern, beweisen die Zugehörigkeit des Minerals zum hexagonalen System.

Bei der Durchsicht eines ganzen Schliffes findet man wohl Stellen, in denen die Orientirung des neuen Minerals eine ganz unregelmässige ist, aber im Grossen und Ganzen ist die Neigung zur Parallelstellung unverkennbar und fuhrt zuweilen zu den vollkommensten Formen. Wie zu erwarten steht, wechseln dagegen die axialen Richtungen $\left(60^{\circ}\right)$ auf kleine Distanzen, so dass eine bestimmte Orientirung der Einlagerungen immer 
nur auf einen mässigen Flächenraum beschränkt ist; es wird dies offenbar durch den umgebenden Albit bestimmt.

Wir sind nunmehr im Stande, diesen mikroskopischen Befund mit den Resultaten der chemischen Untersuchung in Zusammenhang zu bringen. Das die Fasern umschliessende Material ist der Albit; es wird dies, ausser durch die angefuhrten Thatsachen, dadurch bewiesen, dass derselbe, wo er deutlich isolirt ist, die gleiche Structur hat, wie das pseudomorphe Material, ferner durch die fruher erwähnte Untersuchung des löslichen Ruckstandes, aus welchem die Fasern entfernt und die Grundmasse unangegriffen geblieben war. Das eingeschlossene Mineral ist dasjenige, welches mit dem Albit zusammen den $\beta$-Spodumen constituirt, und dessen Zusammensetzung durch die Formel $\mathrm{Li}_{2} \mathrm{Al}_{2} \mathrm{Si}_{2} \mathrm{O}_{8}$ ausgedruckt wird.

In Rucksicht auf die Thatsache, dass dieses Lithiummineral sowohl krystallographisch als chemisch vollkommen definirt ist, und weil es in der Geschichte der Umwandlung des Spodumen eine so wichtige Rolle spielt, glaubten wir demselben einen unterscheidenden Namen geben zu müssen und nannten es nEukryptit ".

Der Eukry p t it krystallisirt hexagonal und besitzt wahrscheinlich basische Spaltbarkeit. Sein spec. Gewicht, berechnet aus dem des $\beta$-Spodumen $=2,647$ und dem des pseudomorphen Albit $=2,637$, ist $=2,667$. Er gelatinirt mit Salzsäure und schmilzt leicht. Seine Zusammensetzung ist die eines Singulosilikates von der Formel $\mathrm{Li}_{2} \mathrm{Al}_{2} \mathrm{Si}_{2} \mathrm{O}_{8}=47,51 \mathrm{SiO}_{2}$, $40,61 \mathrm{Al}_{2} \mathrm{O}_{3}, 11,88 \mathrm{Li}_{2} \mathrm{O}=100$. Seine Beziehungen zu andern Mineralien sind nicht sicher; in Form und chemischer Zusammensetzung ist er analog dem Nephelin; auch könnte man ihn als einen Lithionanorthit betrachten, da er mit diesem Mineral, von welchem er jedoch krystallographisch ganz verschieden ist, das Molekularverhältniss gemeinsam hat. Andererseits könnte die Thatsache, dass er so leicht in Muscovit ubergeht und dasselbe Molekularverhältniss besitzt, wie die normalen Varietăten dieses Minerals, es zu erfordern scheinen, dass man ihn neben diesen stelle; indess besitzt er entschieden keine glimmeräbnliche Structur, und der echte Lithionglimmer (Lepidolith) hat eine sehr abweichende Zusammensetzung.

\section{Cymatolith.}

Dieser Name wurde 1867 von Professor Sh epar d einem zu Goshen und Norwich, Mass., gefundenen Minerale, einem Zersetzungsprodukt des Spodumen, beigelegt. Die von ihm mitgetheilten Analysen liessen die $\mathrm{Zu}-$ sammensetzung des als neu angenommenen Minerals fraglich, und die daruher bestehenden Zweifel wurden auch nicht beseitigt durch eine spätere Analyse von Herrn B. S. Burton. Herr Julien giebt in seiner Abhandlung mehrere Analysen des Cymatolith, welche sowohl unter einander gut ubereinstimmen, als auch einer einfachen chemischen Formel entsprechen. 
Bei unseren ersten Untersuchungen tuber den Gegenstand nabmen wir ebenfalls noch als feststehend an, dass der Cymatolith ein homogenes Mineral sei und eine bestimmte Zusammensetzung besitze; diese Annabme wurde durch zwei sehr gut ubereinstimmende Analysen (siebe unten) des Materials von Branchville bestätigt. Das weitere Studium indess, nothwendig gemacht durch die am $\beta$-Spodumen erhaltenen Resultate (da der Cymatolith direct aus dem letzteren entsteht), hat uns tberzeugt, dass die angenommene Mineralspecies nur ein merkwurdig gleichmässiges und inniges $\mathrm{mech}$ anisches Gemenge von Muscovit und Albit ist. Wir werden indess in dieser Abhandlung fur dasselbe den Namen Cymatolith beibehalten als eine bequeme Bezeichnung für diese interessante zusammengesetzte Substanz und wollen sie zunächst so beschreiben, als ob es eine ächte Mineralspecies wäre.

Die physikalischen Eigenschaften des Cymatolith von Branchville sind die folgenden: deutliche faserige Structur, manchmal gerade, im Allgemeinen jedoch gewunden, zuweilen verworren faserig, endlich auch schuppig; spec. Gew. 2,692-2,699; Farbe meist weiss, aber auch oft schwach gefärbt, so zuweilen mit einem schwachen Stich ins Rothe.

Wie schon S. 192 erwähnt wurde, sind die in Cymatolith umgewandelten Krystalle häufig und oft sehr gross. Die Art, in welcher die Faserstructur entwickelt ist, geht aus Fig. 2, einem Querschnitt senkrecht zur Prismenaxe, hervor. Gewöhnlich ist, wie es die Figur zeigt, die Richtung der Fasern an den Kanten rechtwinkelig zu den Grenzflächen, aber im Inneren ist die Structur eine unregelmässigere, und die Fasern verflechten sich zu einem verworrenen Gewebe, zuweilen von federartigem Ansehen. Gewöhnlich ist jede Spur der ursprunglichen prismatischen Structur und Spaltbarkeit des Spodumens verschwunden; nur selten sieht man noch Etwas davon, wäbrend die Richtung der Fasern auch hier eine transversale bleibt (vergl. auch mehrere andere Figuren auf Taf. IV; $c=$ Cymatolith).

Herr Penfield fuhrte zwei Analysen von Cymatolith aus, von denen $\mathrm{Nr} .1$ mit einem Material angestellt ist, welches von einem ganz umgewandelten Krystall stammt; dasselbe war vollkommen weiss und anscheinend frei von jeder Verunreinigung. Die Resultate sind die folgenden: $\mathrm{Nr}$. 1. Spec. Gew. $=2,692$.

I. II. III. Mittel: Molekularverhältniss :

\begin{tabular}{|c|c|c|c|c|c|c|c|}
\hline $\mathrm{SiO}_{2}$ & 59,38 & - & - & 59,38 & & 0,989 & 4 \\
\hline $\mathrm{Al}_{2} \mathrm{O}_{3}$ & 26,67 & - & - & 26,67 & & 0,259 & 1,05 \\
\hline $\mathrm{CaO}$ & 0,62 & - & - & 0,62 & $0,011)$ & \multirow{4}{*}{0,283} & \multirow{4}{*}{1,13} \\
\hline $\mathrm{Na}_{2} \mathrm{O}$ & - & 7,66 & 7,70 & 7,68 & 0,124 & & \\
\hline $\mathrm{K}_{2} \mathrm{O}$ & - & 3,53 & 3,49 & 3,51 & 0,037 & & \\
\hline $\mathrm{H}_{2} \mathrm{O}$ & 2,01 & - & - & $\frac{2,01}{99,87}$ & $0,111)$ & & \\
\hline
\end{tabular}


Die Substanz zur zweiten Analyse wurde demselben Krystall entnommen, welcher die Portion 2 des $\beta$-Spodumen geliefert hatte, und war ebenfalls ganz rein. Dieselbe ergab folgende Zahlen :

Nr. 2. Spec. Gew. 2,699.

\begin{tabular}{|c|c|c|c|c|c|c|}
\hline \multirow[b]{2}{*}{$\mathrm{SiO}_{2}$} & \multirow{2}{*}{$\begin{array}{c}\text { I. } \\
60,61\end{array}$} & \multirow{2}{*}{$\begin{array}{c}\text { II. } \\
60,49\end{array}$} & \multirow{2}{*}{$\begin{array}{r}\text { Mittel : } \\
60,55\end{array}$} & \multicolumn{3}{|c|}{ Molekularverhältniss : } \\
\hline & & & & & 1,009 & 4 \\
\hline $\mathrm{Al}_{2} \mathrm{O}_{3}$ & 26,37 & 26,39 & 26,38 & & 0,256 & 1,016 \\
\hline $\mathrm{MnO}$ & 0,08 & 0,06 & 0,07 & & \multirow{6}{*}{0,263} & \multirow{6}{*}{1,044} \\
\hline $\mathrm{Na}_{2} \mathrm{O}$ & 8,08 & 8,16 & 8,12 & 0,131 & & \\
\hline $\mathrm{K}_{2} \mathrm{O}$ & 3,33 & 3,35 & 3,34 & 0,035 & & \\
\hline $\mathrm{Li}_{2} \mathrm{O}$ & 0,17 & 0,17 & 0,17 & 0,006 & & \\
\hline $\mathrm{H}_{2} \mathrm{O}$ & 1,65 & 1,66 & 1,65 & $0,091)$ & & \\
\hline & $\overrightarrow{100,29}$ & $\overline{100,28}$ & $\overline{100,28}$ & & & \\
\hline
\end{tabular}

Die Uebereinstimmung zwischen diesen beiden Analysen ist eine so vollkommene, als sie nur erwartet werden kann. Das Molekularverhältniss in $\mathrm{Nr} .2$ ist fast ganz genau

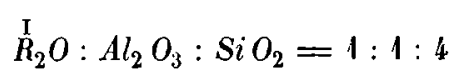

d. b. dasselbe, welches für den Spodumen selbst und für $\beta$-Spodumen gefunden wurde. Die Formel ist demnach

$$
(\mathrm{Na}, \mathrm{K}, \mathrm{H})_{2} \mathrm{Al}_{2} \mathrm{Si}_{4} \mathrm{O}_{12}=\left(\mathrm{K}, \mathrm{H}_{2} \mathrm{Al}_{2} \mathrm{Si}_{2} \mathrm{O}_{8}+\mathrm{Na}_{2} \mathrm{Al}_{2} \mathrm{Si}_{6} \mathrm{O}_{16}\right.
$$

Da der Cymatolith sicher aus dem $\beta$-Spodumen entstanden ist, von dem letzteren aber nachgewiesen wurde, dass er ein Gemenge von Albit und einem Mineral von der Zusammensetzung eines Lithium-Muscovit sei, so ist es bezeichnend, dass die Formel des Gymatolith als diejenige der Verbindung eines Moleküls Muscovit mit einem Molekul Albit geschrieben werden kann. Auch obme andere Thatsachen würde hiernach der Schluss, dass auch der Gymatolith ein mechanisches Gemenge darstellt, kaum angezweifelt werden können, doch liess sich der Beweis dafür auch hier unabhängig von diesen Betrachtungen, in vollkommen sicherer Weise führen. Es muss bemerkt werden, dass die beim $\beta$-Spodumen angewandte chemische Methode in diesem Falle nicht angewendet werden kann, weil der Muscovit durch Salzsäure nicht zersetzt wird. Eine vorläufige Prüfung mit Schwefelsäure ergab eine Angreifbarkeit des Cymatolith, welche sich auf den Glimmer beschränkte, doch wurde diese Methode nicht weiter verfolgt, weil die mikroskopische Untersuchung sich als völlig genügend zur Lösung der in Rede stehenden Frage erwies.

Es wurde eine beträchtliche Zahl von Dunnschliffen, sowohl solche von normalem reinen Cymatolith, als von dessen Uebergängen in $\beta$-Spodumen einer- und Albit andererseits, untersucht und nicht nur nachgewiesen, dass es sich in der That um ein Gemenge von Muscovit und Albit handelt, 
sondern auch die Erklärung fur die auffallende Uebereinstimmung der chemischen Zusammensetzung darin gefunden, dass das Gemenge in den meisten Fällen ein im höchsten Grade inniges ist. Ein Cymatolithschliff, wie er in Fig. 1c dargestellt ist, erscheint im polarisirten Lichte zusammengesetzt aus langen, dunnen, etwas gekrummten Fasern, welche sehr lebhafte Farben geben und die charakteristische Glimmerstructur zeigen, und aus zwischengelagerten graulichgefärbten Partien von Albit. Manchmal sind indess die Glimmerfasern so dichtgedrängt, dass der Albit nicht sichtbar ist ; andere Male sind sie divergent auseinandergespreizt, und alsdann erscheint der aus dem zweiten Mineral gebildete Hintergrund sehr deutlich. An noch anderen Stellen endlich laufen diè mehr vereinzelten Glimmernadeln als glänzende Linien in einer ausgedehnten Albitpartie aus.

Die Schliffe bieten einen um so interessanteren Anblick dar, je unregelmässiger die Structur des Cymatolith ist. Es wurden z. B. zwei Schliffe von dem der Fig. 2 zu Grunde liegenden Krystall hergestellt, von denen einer, wie die Figur einen Querschnitt darstellte, während der andere. senkrecht war und Etwas von der prismatischen Structur des ursprunglichen Minerals erkennen liess. Alle Details des Schliffes erschienen aufs Klarste im polarisirten Lichte, namentlich war dies der Fall mit der Federstructur: es erschien ein tief gefärbter Glimmerstreifen, von welchem aus die schmalen Fasern desselben Minerals nach beiden Seiten regelmässig divergiren, während der Albit zwischen ihnen um so deutlicher zu erkennen ist, je weiter sie von einander abstehen. Ferner wurden Schliffe untersucht von der schuppigen Varietät des Cymatolith, bei welcher die Glimmerschuppen der Oberfläche parallel liegen; in diesen hatte der Albit das bereits beim $\beta$-Spodumen erwähnte gefleckte Ansehen im polarisirten Lichte, und der Glimmer war in lebhaft gefärbten Schuppen sehr gleichförmig in demselben vertheilt. Andere Schliffe, aus deutlich faserigen Exemplaren senkrecht zu der Faserung hergestellt, zeigten wiederum etwas andere Erscheinungen. Es liessen sich noch eine Reibe von Details aufzählen, doch mag das Erwähnte genugen, um diejenigen Erscheinungen zu charakterisiren, auf welche die Feststellung der zusammengesetzten Natur des Cymatolith begrundet ist. Glimmer und Albit sind stets deutlich von einander verschieden und erscheinen zuweilen, indem sie sich beim Process der Umwandlung stellenweise von einander gesondert haben, in grösseren isolirten Partien. (Näheres daruber siehe weiter unten.)

Das einzige sonst noch in den Schliffen beobachtete Mineral bestand aus hexagonalen Prismen, welche kaum etwas Anderes als Apatit sein können, mit dem sie optisch und krystallographisch vollkommen ubereinstimmen. Sie sind durch den Cymatolith, stellenweise ziemlich zahlreich, zerstreut, zuweilen auch im $\beta$-Spodumen, können aber keinen Augenblick mit dem Eukryptit in letzterem verwechselt werden. Die Gegenwart von 
Apatit würde auch den in Analyse 1 des Cymatolith gefundenen Kalkgehalt erklären.

Besonders interessant sind gewisse Schliffe, welche den Uebergang von $\beta$-Spodumen in Cymatolith zeigen. Während bei letzterem vielfach eine Tendenz zu theilweiser Isolirung von Glimmer und Albit zu erkennen ist, giebt es andere Exemplare, in denen beide ebenso innig gemengt sind, wie Eukryptit und Albit im $\beta$-Spodumen. In diesem Falle ist auch die Structur des Cymatolith genau diejenige des $\beta$-Spodumen, nur dass die gerundeten Fasern des Eukryptit durch die dünnen langgestreckten Glimmerschuppen ersetzl sind, deren ganze Erscheinungsweise ihre Bildung aus ersteren beweist. In noch anderen Fällen kann man in einem und demselben Schliff von normalem Cymatolith auf der einen Seite zu normalem $\beta$-Spodumen aur der anderen Seite ubergehen; zwischen beiden Substanzen befindet sich eine Zone, in welcher die Umwandlung des Eukryptit nur eine theilweise ist. Dies wird aus beistehender Fig. 19 ersichtlich werden: diese zeigl. einige Fasern anscheinend unverändert, während andere theilweise umgewandelt sind und zahlreiche dünne Glimmerschuppen, oft dicht zusammengehäuft, enthalten. Diese kleinen Schuppen lassen keine regelmässige Orientirung erkennen, oft liegen sie quer gegen die Fasern des Eukryptit, wie sich sowohl aus der Richtung der Spaltungslinien, als aus der der Auslöschung zwischen gekreuzten Nicols ergiebt. Wo dagegen die Umwandlung eine vollständige ist, liegt die Lamellarstructur des Glimmers im Allgemeinen parallel der Längsrichtung des ursprünglichen

Fig. 19 .

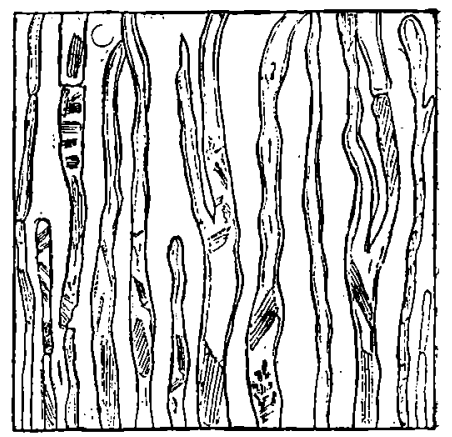

Eukryptit. Die Fasern des letzteren haben in jener Zwischenzone, selbst wenn noch keine Glimmerschuppen zu sehen sind, meist die Schärfe ihrer Umrisse verloren und sind manchmal durch Reihen kleiner, unregelmässiger, durchsichtiger Körnchen gleichsam getheilt.

Der Uebergang von $\beta$-Spodumen in Cymatolith an der Contactlinie ist. auch oft dem blossen Auge erkennbar, indem man die silberglänzenden Glimmerlinien, obgleich deren einzelne Schuppen zu klein sind, um unterschieden zu werden, in den compakten $\beta$-Spodumen eindringen sieht.

\section{Albit.}

Der nach Spodumen pseudomorph auftretende Albit erscheint in mehreren, ziemlich abweichenden Varietäten : entweder ist er feinkörnig ohne deutliche krystallinische Structur, oder faserig, ähnlich wie $\beta$-Spodumen 
und Cymatolith, die Fasern senkrecht zum Prisma; oder er bildet Theile von zersetzten Krystallen und hat dann dieselbe krumme und gewunden blätterige Structur, welche fur den, einen grossen Theil des Ganges bildenden Albit charakteriśtisch ist; endlich erscheint er auch in rosettenförmigen. Aggregaten an der Oberfläche vieler Krystalle eingewachsen in einer Weise, welche deutlich seinen Ursprung als Zersetzungsprodukt erkennen lässt.

Eine Analyse der faserigen Varietät lieferte Herrn Penfield die folgenden Resultate :

Spec. Gew. $=2,637$.

I. II. Mittel : Molekularverhältniss :

\begin{tabular}{|c|c|c|c|c|c|c|}
\hline $\mathrm{SiO}_{2}$ & 67,61 & 67,59 & 67,60 & & 1,127 & 6 \\
\hline $\mathrm{Al}_{2} \mathrm{O}_{3}$ & 20,07 & 20,11 & 20,09 & & 4,195 & 1,03 \\
\hline $\mathrm{MgO}$ & 0,16 & 0,14 & 0,15 & 0,004 & & \\
\hline $\mathrm{Na}_{2} \mathrm{O}$ & 11,71 & 11,66 & 11,69 & 0,188 & 0,193 & 1,02 \\
\hline$K_{2} O$ & 0,11 & 0,11 & 0,11 & 0,001 & & \\
\hline Glühverlust & 0,14 & 0,14 & 0,14 & & & \\
\hline & $\overline{99.80}$ & $\overline{99,75}$ & $\overline{99,78}$ & & & \\
\hline
\end{tabular}

Diese Zahlen entsprechen genau der Formel $\mathrm{Na}_{2} \mathrm{Al}_{2} \mathrm{Si}_{6} \mathrm{O}_{16}$, d. i. derjenigen des Albit.

Das Vorkommen von Albit pseudomorph nach Spodumen erwähnt auch Herr Julien, aber in der Lagerstätte von Massachusetts scheint das Mineral eine verhälınissmässig unbedeutende Rolle zu spielen. Der genannte Autor theilt mit, dass Albit, gemengt mit etwas Muscovit und Quarz, in Pseudomorphosen auftrete, die 》nur eine Varietät " der grobkörnigen Gemenge von Quarz, Feldspath und Glimmer bilden, welche er Pseudomorphosen von »Ganggranit « nennt.

Auf der Lagerstätte von Branchville nimmt der Albit eine wichtigere und vielleicht interessantere Stelle unter den Zersetzungsprodukten des Spodumens ein.

Der faserige Albit, welcher zu der obigen Analyse diente, bildete die ganze Masse eines vollkommen deutlichen Krystalls. Ein aus demselben hergestellter Schliff zeigte inn wesentlich rein mit nur Spuren von Glimmer (siehe den Kaligehalt der Analyse); die Structur war ziemlich undeutlich faserig und, was besonders zu bemerken ist, von demselben Aussehen, wie die des Albit mit dem Glimmer im Cymatolith und mit Eukryptit im $\beta$-Spodumen. Ein Theil der Fasergruppen erwiesen sich im polarisirten Lichte als aus zwei Theilen zusammengesetzt, deren Auslöschungswinkel $10-11^{0}$ mit einander bildeten; da dies der Winkel der Schwingungsrichtungen des Albitzwillings, durch die Basis geseben, ist, so kann diese Uebereinstimmung kaum eine zufällige sein und beansprucht ein gewisses Interesse. . 
Eine Anzahl anderer Schliffe zeigte, dass der reine Albit ziemlich selten ist, und dass die Hauptmenge des körnigen Minerals in den Krystallen eine beträchtliche Menge Glimmer enthält und sich daher dem Cymatolith nähert, ein Punkt, welcher bei der näheren Betrachtung der Taf. IV zu berucksichtigen ist. Manchmal tritt der Albit in breiten Tafeln mit deutlichster Zwillingsbildung auf und mit ihm der Glimmer in Lamellen, welche ebenfalls gross sind, verglichen mit denen des normalen Cymatolith.

\section{Muscovit.}

Als besonderes Mineral, unabhängig von seiner gewöhnlichen Verbindung mit Albit, spielt der Kaliglimmer keine wichtige Rolle unter den Pseudomorphosen des Spodumen von Branchville. Er kommt sehr gewöhnlich als Auskleidung der Bruchflächen im Innern der zersetzten Krystalle vor; ferner findet er sich in kleinen abgesonderten Massen oder in zerstreuten Blättchen innerhalb der Krystallsubstanz selbst. In dieser Weise tritt er besonders auf in den zusammengesetzten Pseudomorphosen, in denen die Feldspäthe (Albit und Mikroklin) ebenfalls in isolirten Partien vorhanden sind; vergl. Fig. 6 und 8 ( $g$-Glimmer). Die Farbe ist gewöhnlich hell grunlich-gelb mit Fettglanz, selten roth, ohne dass desshalb ein Lithionglimmer vorliegt. Es war nicht möglich, eine genügende Menge reinen Materials für eine Analyse zu gewinnen.

Das Vorkommen des Glimmers mit dem Albit gemengt, als Cymatolith, ist bereits unter der Ueberschrift des letzteren beschrieben worden. Die daselbst mitgetheilten Analysen zeigen, dass der Glimmer die Zusammensetzung eines normalen Muscovit, $(\mathrm{K}, \mathrm{H})_{2} \mathrm{Al}_{2} \mathrm{Si}_{2} \mathrm{O}_{8}$, besitzt. Nimmt man das Verhältniss von $\mathrm{K}_{2} \mathrm{O}: \mathrm{H}_{2} \mathrm{O}=1: 3$, wie es angenähert in der Analyse 2 gefunden wurde, so ergiebt sich die berechnete Zusammensetzung dieses Muscovit folgendermassen :

\begin{tabular}{lr}
$\mathrm{SiO}_{2}$ & $\mathbf{4 6 , 2 3}$ \\
$\mathrm{Al}_{2} \mathrm{O}_{3}$ & 39,52 \\
$\mathrm{~K}_{2} \mathrm{O}$ & $\mathbf{9 , 0 5}$ \\
$\mathrm{H}_{2} \mathrm{O}$ & $\mathbf{5 , 2 0}$ \\
\hline & $\mathbf{1 0 0 , 0 0}$
\end{tabular}

Nachdem die zusammengesetzte Natur des Cymatolith einmal festgestellt war, wurden mit Leichtigkeit eine Anzahl Exemplare gefunden, an denen Glimmer und Albit so deutlich waren, dass sie mit unbewaffnetem Auge unterschieden werden konnten. Das Vorkommen von Albit mit geringen Mengen eingelagerten Glimmers wurde schon erwähnt ; umgekehrt fanden wir Stucke, in denen der Glimmer mehr oder weniger vollständig vom Albit getrennt war. Fig. 20 (s. S. 208) zeigt einen Schliff quer durch einen 
Fig. 20.

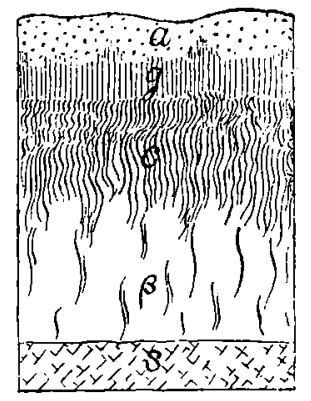

Krystall mit dem Kern von Spodumen $(s)$, dann $\beta$-Spodumen, darauf Cymatolith $(c)$ nit allmäligem Uebergang in reinen und matt silberglänzenden Glimmer $(g)$, endlich eine Decke von Albit. Dieser Fall zeigt, bis zu welchem Grade die Tręnnung der Bestandtheile des Cymatolith gehen kann.

In den Stücken von Massachusetts ist nach Herrn $\mathrm{Ju}$ li e $\mathrm{n}$ isolirt ausgebildeter Glimmer weit massenhafter vorhanden, worüber eine Bemerkung desselben weiterhin mitgetheilt werden soll.

\section{Mikroklin.}

Ein zweites kalihaltiges Mineral, ebenfalls aus dem Spodumen entstanden, ist ein Kalifeldspath von der Zusammensetzung und dem optischen Charakter des Mikroklin, welcher jedoch in den Pseudomorphosen viel seltener vorkommt, als der Albit. Wo er, was selten der Fall ist, allein auftritt, hat er eine feinkörnige Structur und zeigt Nichts von der Spaltbarkeit; seine Farbe ist gelb. Unter dem Mikroskop löst er sich in unabhängige Körner auf, welche das charakteristische Ansehen des Mikroklin im polarisirten Lichte besitzen. An dem besten der untersuchten Exemplare waren die Krystallfächen, prismatische wie Endflächen, vollkommen deutlich, aber von dem ursprünglichen Mineral war Nichts ubrig geblieben; die Pseudomorphose bestand grösstentheils aus dem Kalifeldspath, nur ein kleiner Theil der einen Seite war Natronfeldspath (Albit). Die Beziehung beider Mineralien ist aus Fig. 10 ersichtlich, in welcher ein Fall dargestellt wurde, in dem der Albit in weit grösserer Menge, als in dem zuletzt beschriebenen, vorhanden ist und ungefähr die Hälfte des Krystalls ausmacht.

Die Zusammensetzung des gelben körnigen Feldspathes geht aus der folgenden Analyse des Herrn Penfield hervor:

Spec. Gew. $=2,548$.

\begin{tabular}{|c|c|c|c|c|c|c|}
\hline & I. & II. & Mittel : & \multicolumn{3}{|c|}{ Molekularverhältniss : } \\
\hline $\mathrm{SiO}_{2}$ & \multicolumn{2}{|c|}{ 64,55 } & 64,55 & & 1,076 & 6 \\
\hline $\mathrm{Al}_{2} \mathrm{O}_{3}$ & \multicolumn{2}{|c|}{19,70} & 19,70 & & 0,191 & 1,07 \\
\hline $\mathrm{K}_{2} \mathrm{O}$ & $\longdiv { 1 5 , 6 6 }$ & $\overline{15,59}$ & 15,62 & $0,166\}$ & & \\
\hline $\mathrm{Na}_{2} \mathrm{O}$ & 0,53 & 0,64 & 0,58 & $0,010\}$ & 0,176 & 0,98 \\
\hline GJuhverlust & 0,12 & 0,12 & 0,12 & & & \\
\hline & & & 100,57 & & & \\
\hline
\end{tabular}

Diese Zablen stimmen sehr gut mit der normalen Zusammensetzung des Mikroklin $\mathrm{K}_{2} \mathrm{Al}_{2} \mathrm{Si}_{6} \mathrm{O}_{16}$ überein. 
Fig. 3 und 4 Taf. IV zeigen die Art und Weise, in welcher der Kalifeldspath im Innern des Albit vorkommt, während beide an der Zusammensetzung vollkommen deutlicher pseudomorpher Spodumenkrystalle Theil nehmen. Der Mikroklin findet sich ferner in breiten Spaltungsplatten, zuweilen fast den ganzen Krystall des ùrsprunglichen Minerals bildend; alsdann ist er ebenfalls gewöhnlich mit Albit associirt, wie die Fig. 11 und 13 zeigen. Eine besonders merkwürdige und interessante Thatsache in Rücksicht auf das Zusammenvorkommen beider Mineralien ist die, dass die isolirten Fragmente von Mikroklin, welche an verschiedenen einzelnen Stellen eines und desselben Krystalls zerstreut erscheinen, obgleich manchmal mehrere Zoll von einander entfernt, sich doch sämmtlich in paralleler Stellung befinden; die Richtung der Spaltungsflächen ist jedoch in verschiedenen Krystallen nicht ubereinstimmend, so dass ihre Orientirung zu den Krystallaxen des Spodumens keine bestimmte Beziehung zu besitzen scheint.

Es ist nicht überflussig, bei dieser Gelegenheit mitzutheilen, dass der Mikroklin an der in Rede stehenden Fundstätte in sehr grosser Menge vorkommt; hunderte von Tonnen wurden bereits durch die Herren Smith für die Zwecke der Porzellanfabrikation gewonnen; man fand ibn in Spaltungsplatten von einer Grösse, wie sie kaum zu handhaben waren, und fast rein (eine einzige zusammenhängende Spaltfläche von zehn Fuss Länge wurde auf diesem Lager beobachtet).

\section{Killinit.}

Killinit ist ein wasserhaltiges Kaliumaluminiumsilikat, welches gewöhnlich zur Pinitgruppe gestellt wird; es wurde zuerst von der KillineyBay in Irland beschrieben und zwar als ein zusammen mit Spodumen, dessen Spaltbarkeit es besitzt, in Granit vorkommendes Mineral ; eine Anzahl Analysen desselben wurden in Greg und Let ts o m's Mineralogie mitgetheilt. Dasselbe Mineral beschrieb Herr Juli en als in Chesterfield, Massachusetts, vorkommend, und seine Resultate werden grösstentheils von den nunmehr mitzutheilenden unsrigen bestätigt.

Der Killinit von Branchville ist manchmal dicht und structurlos, meistens jedoch undeutlich faserig parallel dem Prisma des ursprünglichen Minerals, des Spodumen, dessen Spaltbarkeit oft noch deutlich zu erkennen ist. Die Farbe wechselt zwischen verschiedenen Nuancen von Grün, von licht bläulich grün bis ölgrun und dunkel grasgrün.

Es wurden zwei Analysen mit verschiedenem Material ausgeführt: Nr. 1 von der prismatischen Varietăt durch Herrn S. L. Penfield, Nr. 2 von dem dichten Mineral durch Herrn F. P. Dewey.

Groth, Zeitschrift f. Krystallogr. V. 


$\begin{array}{lrr} & \mathrm{Nr} .1 . & \mathrm{Nr} .2 . \\ \mathrm{SiO}_{2} & 48,93 & 53,47 \\ \mathrm{Al}_{2} \mathrm{O}_{3} & 34,72 & 32,36 \\ \mathrm{Fe}_{2} \mathrm{O}_{3} & 0,54 & 0,79 \\ \mathrm{FeO} & 0,33 & 0,42 \\ \mathrm{MnO} & 0,64 & 0,72 \\ \mathrm{CaO} & - & 0,17 \\ \mathrm{~K}_{2} \mathrm{O} & 9,64 & 7,68 \\ \mathrm{Na}_{2} \mathrm{O} & 0,35 & 0,44 \\ \mathrm{Li}_{2} \mathrm{O} & - & 0,04 \\ \mathrm{H}_{2} \mathrm{O} & 5,04 & 4,07 \\ & \mathbf{1 0 0 , 1 9} & \frac{100,16}{}\end{array}$

Die beiden Analysen zeigen eine ziemlich beträchtliche Differenz in der chemischen Zusammensetzung der beiden Varietäten. Vergleicht man damit und mit einander die von Greg und Lettsom publicirten, sowie die von Herr Julie n mitgetheilten Analysen, so ergieht sich, dass die $\mathrm{Zu}-$ sammensetzung des Minerals in sehr weiten Grenzen schwankt. Es ist indess kein Zweifel, dass in den verschiedenen Fällen doch im Wesentlichen dasselbe Material zur Untersuchung gedient hat, und dass die gefundenen Differenzen wahrscheinlich durch Inhomogenität der Substanz verursacht sind.

Der Killinit giebt im gèschlossenen Rohr Wasser; vor dem Löthrohr erglubt er und schmilzt etwa bei 5 zu einem weissen Email. Nicht zersetzbar durch Salzsäure.

Es wurden verschiedene Dunnschliffe des Killinit mikroskopisch untersucht; in denselben zeigte sich deutlich die parallelfaserige Structur und ausserdem erschienen Blättchen zu beiden Sejten jeder parallelen Linie unter gleichen Winkeln in entgegengesetzter Richtung angelagert; dieselben wirkten lebhaft auf das polarisirte Licht. Während die meisten Präparate eine so feine Structur besassen, dass sie keine befriedigende mikroskopische Analyse gestatteten, schien ein etwas grobkörnigerer Schliff zum Ziele zu fuhren. Derselbe zeigte sich grösstentheils zusammengesetzt aus winzigen Schuppchen, welche alle Eigenschaften des Glimmers besassen und auffallend ähnlich dem unzweifelhaften Glimmer waren, welcher aus der Zersetzung des Eukryptit gebildet und in Fig. 19 dargestellt ist. Hiernach scheint kaum ein Zweifel, dass der Killinit in der Hauptsache aus Glimmer besteht, neben dessen Lamellen kleine, nicht polarisirende Partikel, vielleicht amorphe Kieselsäure, und gelegentlich noch andere, weniger leicht zu bestimmende, vorkommen.

Die Idee einer Beziehung der Mineralieh der Pinitgruppe zu denjenigen Kaliglimmern, welche bei der Analyse Wasser liefern, ist keine neue, sondern ist schon vor langer Zeit ausgesprochen worden; sie wurde erwähnt 
von J. D. D ana in der 5. Ausg. seines Syst. of Min. (1868), S. 447. Ein Vergleich der Analyse 1 des Killinit mit der des Muscovit (S. 207) und mit den älteren Muscovitanalysen (s. D a na's Min.) lässt die Beziehung sofort hervortreten. Die Abweichung der zweiten Killinitanalyse erklärt sich leicht durch die Annahme der Gegenwart einiger Procente freier Kieselsäure, von welcher abgesehen die Uebereinstimmung eine ganz gute ist; zudem haben, wie oben erwähnt, die mikroskopischen Beobachtungen unabhängig davon zu der Vermuthung einer wahrscheinlichen Anwesenheit von amorpher Kieselsäure gefuhrt. Die Rolle, welche der Glimmer uberhaupt in dem Umwandlungsprocess des Spodumen spielt, macht gewiss ebenfalls die obige Ansicht uber die Natur des Killinit wahrscheinlich, obgleich eine definitive Formel für denselben wegen seines Mangels an Homogenität nicht gegeben werden kann.

\section{Pseudomorphosen von Ganggranit.}

Wir bedienen uns desselben Namens, wie Herr J u li en, zur Bezeichnung gewisser pseudomorphosirter Spodumenkrystalle, welche aus einem mehr oder weniger grobkörnigen Aggregat von Feldspath (Albit und Mikroklin) und Glimmer bestehen. In diesen Pseudomorphosen, welche bei Branchville seltener zu sein scheinen; als bei Chesterfield, sind die sie zusammersetzenden Mineralien deutlich ausgebildet und zwar so, wie in der eigentlichen Gangmasse; der Feldspath z. B. ist nicht körnig und anscheinend ohne Spaltbarkeit, wie er es in der Mehrzahl der oben beschriebenen Fälle ist, sondern tritt ziemlich grossblätterig auf. Die Oberfläche dieser umgewandelten Krystalle ist sebr rauh, oft mit rosettenförmigen Albitaggregaten bedeckt, doch lässt sich die allgemeine Form des ursprünglichen Spodumen stets noch deutlich erkennen. Bemerkt zu werden verdient, dass Quarz in diesen zusammengesetzten Pseudomorphosen meist gänzlich fehlt, wodurch sich dieselben wesentlich von denen von Chesterfield unterscheiden.

\section{Beziehungen zwischen den verschiedenen Zersetzungsprodukten des Spodumen in Rücksicht auf ihr Vorkommen.}

Im Folgenden sollen der speciellen Charakterisirung der einzelnen aus dem Spodumen hervorgegangenen Mineralien und dem bereits uber ihre gegenseitigen Beziehungen Gesagten noch einige mehr allgemeine Bemerkungen uber die Art ihres Vorkommens hinzugefügt werden.

Spodumen und $\beta$-Spodumen. Die Art, wie diese beiden Mineralien zusammen vorkommen, ersieht man am besten aus Fig. 5 : das Zersetzungsprodukt, der $\beta$-Spodumen, bildet eine mehr oder weniger dicke Rinde um das ursprungliche Mineral und dringt ausserdem in dasselbe ein in Form von Streifen, welche den Richtungen der Spaltungsflächen folgen 
und theils ganz dunn, theils aber auch von beträchtlicher Dicke sind. Es ist bemerkenswerth, dass in allen Fällen die Trennungslinie zwischen beiden vollkommen scharf ist, und dass der so vorkommende Spodumen selten und böchstens eine Spur von Zersetzung zeigt. Stets ist, wie schon erwähnt, die Richtung der Fasern senkrecht zu der nächsten Oberfläche des Spodumens, doch ist manchmal das Mineral so dicht, dass es nur undeutliche Structur zeigt.

Einen sehr interessanten Fall bot ein zum Theil aus $\beta$-Spodumen, zum Theil aus dem ursprunglichen Mineral bestehender Krystall dar, indem das letztere, obgleich in anderen Beziehungen unverändert, bereits die Transversalstructur des ersteren angenommen hatte, und die longitudinale prismatische Structur fast ganz verwischt war. Es ist dies offenbar die erste Stufe in dem Umwandlungsprocess.

$\beta$-Spodumen und Cymatolith. Viele Krystalle und Fragmente solcher, welche keine Spur von Spodumen zeigen, lassen die Beziehungen dieser beiden Substanzen in sehr deutlicher Weise erkennen. Der Anblick derselben lässt keinen $Z$ weifel darüber, dass die erstere allmälig in die zweite ubergeht, indem die Blättchen des Cymatolith mit den weniger deutlichen Fasern des $\beta$-Spodumen verflochten sind, überall wo beide in Contact treten (s. Fig. lb); dabei ist ihr Ansehen jedoch immer dasjenige zweier verschiedener Substanzen, selbst wenn die Beruhrungslinie unter dem Mikroskope untersucht wird. Dies fuhrt zu dem Schlusse, dass, während die Umwandlang allmälig vor sich gegangen ist, in chemischer Beziehung ein rascher Wechsel von der einen Substanz zur andern stattfindet. Wie früher bemerkt, wurde die Analyse 2 des $\beta$-Spodumen mit einem Material ausgefuhrt, welches, obgleich anscheinend rein, unmittelbar an den $\mathrm{Cy}-$ matolith angrenzte, und da das Resultat mit den ubrigen harmonirte, so kann ein allmäliger Uebergang nicht angenommen werden. Viele der grossen Cymatolithkrystalle zeigen bei sorgfältiger Untersuchung eine Spur des andern Minerals, und wir glauben annehmen zu müssen, dass dasselbe wenigstens an dieser Lagerstätte stets vorhanden war.

Spodumen, $\beta$-Spodumen und Gymatolith. Es erubrigt noch, von denjenigen Fällen zu sprechen, in denen alle drei Mineralien zusammen in demselben Krystall erscheinen. Mit Uebergehung vieler anderer Beispiele wird es genügen, einen besonders auffallenden Fall zu beschreiben: die Figuren 1a, 1b, 1c stellen drei Querschnitte eines und desselben Krystalls dar, welcher eine Länge von 15 Zoll, eine Breite von $4 \frac{1}{2}$ und eine Dicke von 1 Zoll hatte und an dem einen Ende gut ausgebildet war. Die Schliffe, genommen in der Reihenfolge vom ausgebildeten Ende nach abwärts, theilen den Krystall in drei annähernd gleiche Theile. Nr. I (Fig. 1a) zeigt den $\beta$-Spodumen ( $\beta$ ) die Hauptmasse des Krystalls bildend und einen Streifen von unverändertem Spodumen an der unteren Seite, 
während der Cymatolith $(c)$ das Ganze als eine düne, mehr oder weniger zusammenhängende Rinde umgiebt. Nr. 2 (Fig. 1b) enthält keinen Spodumen mehr, sondern der $\beta$-Spodumen bildet den grössten Theil des Querschnittes; indess hat die Menge des Cymatoliths gegenuber dem ersten Schliffe zugenommen. Nr. 3 (Fig. 1c) zeigt nur Cymatolith.

Der bereits oben besprochene, in Fig. $\breve{b}$ dargestellte Schnitt enthält alle drei Mineralien, den Cymatolith jedoch nur sparsam und zwar an den Kanten. In Fig. 8 und 14 bildet der Spodumen einige Punkte und kleine isolirte Partien, deren Umriss den Spaltungsflächen parallel ist; er wird von $\beta$-Spodumen und Cymatolith umgeben, deren letzterer eine von den Spodumencentren ausgehende Radialstructur besitzt. Krystalle, welche nur aus Spodumen und Gymatolith bestehen, sind selten. In Fig. 14 sieht man einen einzigen schmalen Streifen, welcher noch aus $\beta$-Spodumen zusammengesetzt ist, während der ganze ubrige Krystall in Cymatolith und Albit umgewandelt ist.

Albit und Cymatolith. Wie bereits erwähnt wurde, ist der Albit sehr verbreitet in den in Rede stehenden Pseudomorphosen. Während er manchmal fast den ganzen Krystall bildet, tritt er andere Male nur in kleinen isolirten Partien auf. Gewöhnlich ist er feinkörnig, aber auch faserige Structur ist nicht selten. Es ist noch daran zu erinnern, dass zwischen dem normalen Cymatolith (1 Mol. Albit +1 Mol. Muscovit) und dem reinen Albit einerseits, sowie dem reinen Muscovit andererseits viele Uebergänge existiren.

Figur 3 stellt den verticalen Durchschnitt durch einen Theil eines Krystalls dar, dessen Rinde aus faserigem Cymatolith $(c)$ und das Innere aus körnigem Albit $(a)$ mit einigen Streifen von Mikroklin $(m)$ besteht. Fig. 7 ist der Querschnitt eines grossen Krystalls, in welchem beide Mineralien ähnlich vertheilt sind. Fig. 10, 11 und 13 zeigen Albit und Mikroklin, Fig. 6, 8 und 14 körnigen Albit und Cymatolith, sowie die Art, in welcher dieselben gemengt erscheinen. In anderen Fällen tritt der Albit in breiten gekrümmten Platten auf, welche Nichts von einem pseudomorphen Charakter zu haben scheinen bis auf die noch deutlich erhaltene Spodumenform der Krystalle, von welchen sie einen grösseren oder geringeren Theil bilden.

Vorkommen von Killinit und Gymatolith. In vielen der derben Stucke ist der Spodumen zum Theil in Killinit, zum Theil in Cymatolith verändert, und diese beiden Mineralien sind innig mit einander verbunden. Diese Association ist in Fig. 9 dargestellt, in welcher $c$ den faserigen Cymatolith und $k$ den Killinit bedeutet. Der letztere bildet die Hauptmasse des Stuckes, während der Cymatolith mit seiner gewöhnlichen transversalen Faserstructur in dünnen Bändern auftritt, welche nahezu den ursprunglichen Spaltungsrichtungen folgen. 
Es ist die verworren krystallinische derbe Varietät des Spodumen, welche fast den gesammten Killinit geliefert hat; in den deutlichen Krystallen erscheint er selten und zwar manchmal als eine mehr oder weniger unregelmässige Oberflächenschicht, wie in Fig. 12.

\section{Genetische Beziehung zwischen dem ursprünglichen Spodumen und seinen verschiedenen Zersetzungsprodukten.}

Der allgemeine Charakter des Processes, durch welchen der Spodumen in die verschiedenen Umwandlungsprodukte ubergeht, besteht wesentlich aus einer Ersetzung des Lithium durch Natrium und Kalium. Die im Vorbergehenden beschriebenen interessanten Thatsachen uber die zusammengesetzte Natur der mit den Namen $\beta$-Spodumen und Cymatolith belegten Substanzen und das Vorkommen des Muscovit und der beiden Feldspäthe machen den ganzen Process leidlich klar und einfach. Dass zwei Moleküle Spodumen durch eine Subștitution in den Alkalien je ein Molekül Muscovit. und Albit liefern können, wurde bereits von Herrn Julien dargelegt und dazu benutzt, das Vorkommen derjenigen Pseudomorphosen zu erklären, welche aus deutlich individualisirtem Albit und Muscovit bestehen. Das Studium der Exemplare von Branchville setzt uns in den Stand, diese Erklärung zu erweitern und zu vervollständigen.

Die Beziehungen der'verschiedenen Mineralien der Umwandlungsreihe, welche durch den Wechsel in den Alkalien aus dem Spodumen hervorgeht, sind aus folgender Tabelle zu ersehen:

$$
\begin{aligned}
& 2\left[\mathrm{Li}_{2} \mathrm{Al}_{2} \mathrm{Si}_{4} \mathrm{O}_{12}\right]=\left[\mathrm{Li}_{2} \mathrm{Al}_{2} \mathrm{Si}_{2} \mathrm{O}_{8}+\mathrm{Na}_{2} \mathrm{Al}_{2} \mathrm{Si}_{6} \mathrm{O}_{16}\right], \beta \text {-Spodumen } \\
& \text { Spodumen. Eukryptit. Albit. } \\
& =\left[(\mathrm{K}, \mathrm{H}\rangle_{2} \mathrm{Al}_{2} \mathrm{Si}_{2} \mathrm{O}_{8}+\mathrm{Na}_{2} \mathrm{Al}_{2} \mathrm{Si}_{6} \mathrm{O}_{16}\right], \text { Cymatolith } \\
& \text { Muscovit. Albit. } \\
& =(\mathrm{K}, \mathrm{H})_{2} \mathrm{Al}_{2} \mathrm{Si}_{2} \mathrm{O}_{8}+\left\{\mathrm{Na}_{2} \mathrm{Al}_{2} S i_{6} \mathrm{O}_{16},\right. \text { Albit. } \\
& \text { Muscovit. } \quad \text { oder } \mathrm{K}_{2} \mathrm{Al}_{2} \mathrm{Si}_{6} \mathrm{O}_{16} \text {, Mikroklin. }
\end{aligned}
$$

Die erste Stufe des Processes war die Bildung des $\beta$-Spodumen durch Substitution von Natrium für die Hälfte des Lithiums und durch Zerfallen der ursprtinglichen Zusammensetzung in gleiche Moleküle Albit und Eukryptit. Das neue Mineral, welches wir mit diesem letzteren Namen belegten und weiter oben ausführlich charakterisirten, scheint eine verhältnissmässig unbeständige Verbindung zu sein, da es sich so- leicht in Muscovit umwandelt.

Der zweite Theil der Umwandlung war die Bildung des Cymatolith aus. dem $\beta$-Spodumen; dieselbe erfolgte durch den Eintritt von Kalium (und Wasserstoff) fur das noch zurickgebliebene Lithium im Eukryptit und die hieraus folgende Entstehung von Muscovit. Das Resultat war somit ein 
Gemenge gleicher Molekule Albit und Muscovit. Es ist hierbei gewiss das Auffallendste an dieser zusammengesetzten Substanz, dass dieselbe von verschiedenen Fundorten eine so ubereinstimmende chemische Zusammensetzung zeigt. Die Erklärung hierfür ist in der Natur des chemischen Processes zu suchen, durch welchen die Veränderung vor sich ging: die Reaction schritt gleichmässig durch die ganze Masse vor und, wenigstens in den meisten Fällen, ohne eine deutliche Trennung der beiden gebildeten Constituenten. Die Bildung des $\beta$-Spodumens muss durch die Einwirkung einer natronhaltigen, die des Cymatolith durch eine kalihaltige Lösung erfolgt sein.

Es wäre nun noch zu sprechen von denjenigen Pseudomorphosen, in welchen Glimmer und Feldspath in erkennbarer Form und nicht, wie es meist der Fall ist, als unauflösbares Aggregat auftreten. In Bezug auf den Cymatolith wurde bemerkt, dass in seinen gewöhnlichen Varietäten das Gemenge von Muscovit und Albit ein ausserordentlich inniges und gleichmässiges war; dies ist der gewöhnliche Fall bei normalem Material, wie es in Hunderten von Exemplaren gewonnen wurde. Es giebt indess andere, für welche dies nicht gilt; bei denen entweder der vom Glimmer herrührende silberartige Glanz mehr oder weniger fehlt, und deren Substanz sich dem reinen Albit nähert, oder wo im Gegentheil der Albit fast ganz zurücktritt und der Glimmer fast rein erscheint (s. Fig. 20). Diese Thatsachen haben uns zu dem Schlusse gefuhrt, dass es zwischen normalem Cymatolith und reinem Albit und Muscovit zahlreiche Uebergangsstufen giebt, in denen eine ausgesprochene Absonderung der beiden Mineralien eingetreten ist. Eine besondere Erklärung erfordern diese Fälle nicht, denn es ist nicbt nur einzusehen, dass die Bedingungen solche waren, wie sie gelegentlich zu einer derartigen Trennung fuhren mussten, sondern es ist im Gegentheil auffallend, dass dieselbe verhältnissmässig selten war und dass der normale Cymatolith die Regel bildet.

Das oben aufgestellte Schema erfordert offenbar, dass Muscovit und Albit sich zu gleichen Molekulen, d. h. im Gewichtsverhältniss von nahe $1: 2$, gebildet haben. Eine Frage entsteht in Bezug hierauf durch die nicht selten zu beobachtende Abwesenheit des Glimmers in grossen Massen von Albit. Allerdings erscheint derselbe isolirt als schuppiger Ueberzug auf den Bruchflächen der zersetzten Krystalle und hie und da in kleinen abgesonderten Massen, aber die so vorkommende Quantität ist viel geringer, als die Gleichung erfordert. Wir sind demnach zu dem Schlusse gezwungen, dass entweder der gleichzeitig mit dem Albit gebildete Glimmer vollständig verschwunden ist, oder dass die Art der Bildung des Albit zuweilen von der oben auseinandergesetzten verschieden ist. Es kann kaum eine Frage sein, dass derselbe in den erwähnten Fällen unabhängig von seinem Begleiter, dem Muscovit, entstanden sein muss. Wo der Albit eine deutliche Faserstructur besitzt, kann er nur aus dem $\beta$-Spodumen entstanden sein 
(vergl. auch Fig. 8 und 12), und zwar wabrscheinlich durch die Einwirkung. der Lösung eines Natriumsilikates, welche das Lithium des Eukryptit durch Natrium ersetzte und zwei Molekule Kieselsäure einfuhrte. Ferner mag er anch unmittelbar aus dem Spodumen entstanden sein, wie es die folgende Gleichung ausdruckt :

$$
\begin{gathered}
\mathrm{Li}_{2} \mathrm{Al}_{2} \mathrm{Si}_{4} \mathrm{O}_{12} \\
\text { Spodumen. }
\end{gathered}
$$

Ausser den Albitpseudomorphosen kommen auch solche vor, welche aus Kalifeldspath, Mikroklin, bestehen und ebenfalls eine Erklärung erheischen. In denselben ist, wie bereits angegeben, entweder körniger Mikroklin in Albit eingeschlossen, oder ersterer bildet im Wesentlichen den ganzen Krystall, oder endlich er ist in grossen Lamellen in demselben zerstreut. Die S. 214 gegebene Formel (3) zeigt, dass die für Albit und Muscovit gegebene Formel auch dem Mikroklin und Muscovit entspricht, mit den einzigen Unterschied, dass das für das Lithium eintretende Alkali ein anderes ist. Im Allgemeinen fehlt indess der Glimmer, und wir mussen also dann, wie beim Albit, jedoch mit Berucksichtigung des Wechsels im Alkali, schreiben:

$$
\begin{gathered}
\mathrm{Li}_{2} \mathrm{Al}_{2} \mathrm{Si}_{4} \mathrm{O}_{12}+2 \mathrm{SiO}_{2}=\underset{\text { Spodumen. }}{\mathrm{K}_{2} \mathrm{Al}_{2} \mathrm{Si}_{6} \mathrm{O}_{16}} \text { Mikroklin. }
\end{gathered}
$$

Es liegt kein Grund vor, anzunehmen, dass der Mikroklin nicht direct aus dem Spodumen entstanden wäre ; auch wurde keine. Spur einer Kaliverbindung beobachtet, welche etwa dem $\beta$-Spodumen und.Cymatolith entsprochen hätte.

Finige Bemerkungen sind noch hinzuzufügen betreffend die Erklärung der grobkörnigen zusammengesetzten Pseudomorphosen, welche aus Glimmer und beiden Feldspäthen besteben. Die Art ihrer Bildung ist in der bereits besprochenen mit einbegriffen, mit dem einzigen wesentlichen Unterschiede, dass die dabei obwaltenden Bedingungen statt einer innigen Mischung der resultirenden Mineralien eine Absonderung und gleichzeitige Krystallisation derselben in grösseren Massen hervorgebracht haben. In Bezug auf diese Pseudomorphosen ist noch einmal auf die, jedenfalls mit den Bedingungen ihrer Bildung zusammenhängende Thatsache hinzuweisen, dass die Mikroklinpartien eines und desselben Krystalls, obgleich oft isolirt und scheinbar ganz unabhängig von einander, doch in den meisten Fällen sich in paralleler Orientirung befinden.

Diese zusammengesetzten Pseudomorphosen scheinen nach der Beschreibung des Herrn Julie $\mathbf{n}$ an den Fundorten von Massachusetts häufiger $\mathrm{zu}$ sein, als an dem unsrigen. Indess unterscheiden sie sich dort von denen von Branchville dadurch, dass sie viel mehr Glimmer und auch Quarz enthalten; welches letztere Mineral bei den von uns beschriebenen wirklich. 
fehlt. Ausser seiner allgemeinen Erklärung der Beziehung des Natronfeldspath und Muscovit zu dem ursprünglichen Spodumen (welche wir oben, wie erwähnt, angenommen haben) lenkt Herr Jul i en die Aufmerksamkeit noch auf die Thatsache, dass der Spodumen durch Austausch des Alkali und Verlust zweier Molekủle Kieselsäure Muscovit zu liefern im Stande ist, wie die folgende Gleichung zeigt :

$$
\underset{\text { Spodumen. }}{\mathrm{Li}_{2} \mathrm{Al}_{2} \mathrm{Si}_{4} \mathrm{O}_{12}}=\underset{\text { Muscovit. }}{\left(\mathrm{K}, \mathrm{H}_{2} \mathrm{Al}_{2} \mathrm{Si}_{2} \mathrm{O}_{8}+2 \mathrm{SiO}_{2}\right.}
$$

Nach seiner Ansicht ist der in den Pseudomorphosen vorkommende freie Quarz ein deutlicher Beweis dafür, dass dieser Process wirklich stattgefunden hat.

In Beziehung hierauf von Interesse ist die folgende Bemerkung des Herrn Ju li e n: "Viele Pseudomorphosen wurden in dem Gange von Chesterfield gefunden, welche zum grossen Theil oder ganz aus einem grünlich gelben Muscovit von eigenthumlichem Fettglanz bestehen. In der That wurden alle Grade der Beimengung desselben zum Cymatolith beobachtet, von den fast reinen Pseudomorphosen des letzteren Minerals, in welchen der Muscovit nur in kleinen und selbst mikroskopischen Schuppen vorkommt, welche meist parallel zur Verticalaxe des Krystalls liegen - bis zu solchen, in denen der Glimmer so vorherrscht, dass er dem Gemenge eine gelbe ',der grünliche Farbe verleiht, und schliesslich reinen Glimmerpseudomorphosen, vollkommen frei von Cymatolith und dennoch die Form und die Oberflächenstreifung des Spodumen selbst an den Endflächen noch bewahrend."Ferner spricht derselbe von dem Vorkommen grosser Quantitäten von Quarz in den Pseudomorphosen.

In dem von uns untersuchten Material ist es gerade umgekehrt; der Quarz fehlt fast ganz, der Glimmer kommt für sich nur sparsam vor, und zur Bildung der beiden Feldspäthe hat oft die Aufnahme von Kieselsäure aus irgend einer ausserhalb liegenden Quelle stattgefunden.

Schliesslich bleibt noch ein wichtiges pseudomorphes Mineral zu besprechen ubrig, der Killinit. Es scheint uns nicht geeignet, diesem einen bestimmten Platz in einem Schema von der Art des weiter oben mitgetheilten zu ertheilen, aus dem Grunde, weil seine wahre Zusammensetzung noch einigermaassep zweifelhaft ist, und er sicher ein mebr oder weniger unreines Material darstellt, welches denselben Mangel an Homogenität und bestimmter Zusammensetzung besitzt, wie er so häufig unter den Mineralien der Pinitgruppe beobachtet wird. Die mikroskopische Structur und nicht minder die Resultate der Analyse scheinen unsere Vermuthung zu rechtfertigen, dass der Killinit im Wesentlichen ein wasserstoffhaltiger Kaliglimmer ist, nicht sehr verschieden von dem im Cymatolith enthaltenen. In diesem Falle hat sein Auftreten nichts Auffallendes, indem dann die Unterseheidung zwischen ihm und dem letzteren mehr auf der Art der 
Ausbildung, als der chemischen Zusammensetzung beruht. Der chemische Process, welcher zur Bildung des Killinit fuhrt, ist insofern klar, als derselbe wesentlich in der Einfuhrung von Kalium und Wasserstoff an die Stelle von Lithium und in einem Verlust an Kieselsäure besteht. Er kann daher durch die oben angegebene Gleichung (6) ausgedrukkt werden, und die dabei frei gewordene Kieselsäure mag ihrerseits bei der Bildung des Albit eine Rolle gespielt haben (s. Gleichung 4). Es ist erwähnenswerth, dass der Killinit sich wahrscheinlich stets unmittelbar aus dem ursprünglichen Spodumen gebildet hat, da er so gewöhnlich dessen Spaltungsflächen noch erkennen lässt.

Allgemeine Uebersicht. Die in den vorhergehenden Paragraphen gemachten Bemerkungen können zu dem Folgenden zusammengefasst werden : - Der Spodumen war der Einwirkung von natron-, respective kalihaltigen Lösungen unterworfen. Die erste Einwirkung der natronhaltigen Solution fuhrte durch theilweise Ersetzung des Alkali's zu der Bildung einer scheinbar homogenen, in Wirklichkeit jedoch zusammengesetzten Substanz, welche aus gleichen Molekulen Albit und eines neuen Lithiumsilikates (Eukryptit) besteht. Eine fernere Wirkung derselben Lösung (der eines Natronsilikates) lieferte in einigen Fällen, durch vollständigen Austausch des Kalium und gleichzeitige Aufnahme von Kieselsäure, Albit. Auf der anderen Seite trat bäufiger ein Einfluss der kalihaltigen Lösung in der Art ein, dass das erwähnte Lithiumsilikat in normalen Muscovit umgewandelt wurde, und so eine andere scbeinbar homogene, in Wirklichkeit aber ebenfalls zusammengesetzte Substanz, der Gymatolith, resultirte, bestehend aus Muscovit und Albit in gleichem Molekularverhältniss; an Stelle dieses bildete sich, wenn eine Trennung der genannten beiden Mineralien eintrat, ein Gemenge isolirter Partieen Albit und Glimmer. Ferner fubrte die Einwirkung des Kali's, unter Austausch des Alkali und gleichzeitiger Aufnahme von Kieselsäure, zu der Bildung von Kalifeldspath oder Mikroklin; in einigen Fällen war das Resultat ein grobkörniges Gemenge von Glimmer und von beiden Feldspäthen. Eine noch andere Wirkung der Kalilösung bestand endlich, verbunden mit einem Verlust von Kieselsäure, in der Bildung eines dem Glimmer sehr nahestehenden Minerals, des Killinit, direct aus dem ursprünglichen Spodumen.

Es erheben sich hier zwei Fragen, auf welche eine recht befriedigende Antwort zu geben wir nicht im Stande sind. Die erste betrifft die Quelle des Natrium und Kalium, welche in den erwähnten Umwandlungen als Agentien wirkten, und hiervon kann man nur sagen, dass sie wahrscheinlich durch die vorhergehende Zersetzung von Feldspäthen geliefert wurden, ohne dass es möglich ist, die näheren Ưmstände zu bezeichnen.

Die andere Frage ist der schliessliche Verbleib des aus dem Spodumen entfernten Lithium, welches vollkommen verschwunden zu sein scheint, 
wenn man dasselbe nicht etwa als Ursache dafür ansehen will, dass ein Theil des im Gange auftretenden Biotits lithionhaltig ist. Dazu kommt noch, dass der Mangantriphylin, der Lithiophilit, sicher ein ursprügliches Gangmineral.ist und ziemlich häufig mit dem derben Spodumen vorkommt; seine Zersetzung hat den in Rede stehenden Vorrath an Lithium noch vermehrt. Allerdings ist es wahrscheinlich, dass die Bildung der merkwurdigen Reihe von Manganphosphaten, welche wir von dieser Fundstätte beschrieben haben, mit den ausgedehnten Umwandlungsprocessen des Spodumen im Zusammenhange steht. Die Thatsache, dass zwei dieser Phosphate durch ihren Alkaligehalt in dieser Gruppe fast einzig dastehen (s. die Analysen von Dickinsonit und Fillowit in unseren fruheren Abhandlungen), scheint dies fast zu beweisen. Es mag dann der Lithiophilit als das ursprüngliche Manganphosphat, aus welchem die anderen entstanden sind, anzusehen sein, ein Punkt, auf welchen wir in einem späteren Aufsatze wieder zuruckkommen werden.

Im Folgenden sollen noch einige nachträgliche Bemerkungen mitgetheilt werden.

Der Cymatolith ist oft ausser der bereits beschriebenen einer weiteren Umwandlung unterworfen worden, deren Resultat die Bildung eines weichen seifenartigen weissen, mit Glimmerschuppen gemengten Minerals ist, offenbar eines unreinen Kaolin. Dies ist nicht uberraschend, da bekanntlich der Kaolin sowohl aus Natronfeldspath, als aus Kalifeldspath entstehen kann, und da der feinvertheilte Zustand, in welchem der Albit im Cymatolith existirt, ihn besonders leicht der zur Bildung von Kaolin fuhrenden, leicht verständlichen Umwandlung zugänglich machen muss.

Zusammen mit dem weichen kaolinisirten Cymatolith kommt ein interessantes fleischrothes, thonähnliches, dem Montmorillonit nahestehendes Mineral vor. Dasselbe überzieht zuweilen auch die Spaltungsflächen der theilweise zersetzten Spodumenkrystalle, am massenhaftesten findet es sich jedoch in besonderen Ablagerungen in dem Gange, nicht weit von der Stelle, wo der Spodumen vorkommt. Es bildet weiche, leicht mit dem Spaten auszugrabende Massen, von denen an einer Stelle genug gefunden wurde, um einen gewöhnlichen Karren damit zu füllen. Ferner durchdringt es auch die Gangmasse derart, dass es Hohlräume im unzersetzten Albit und Quarz ausfüllt. Es ist sehr unrein, oft stellenweise durch Manganoxyd gefärbt, und enthält Krystalle von Apatit.

Frisch an die Luft gebracht, war es feucht und weich, leicht zwischen den Fingern zerreiblich und in den reinsten Partieen ganz frei von sandigen Beimengungen; einige Wochen der Luft ausgesetzt verlor es viel von seiner Feuchtigkeit und wurde beträchtlich bärter. 
Die Farbe ist ein zartes Rosenroth, wird aber an'der Luft allmälig etwas blasser. Vor dem Löthrohr schmilzt die Substanz leicht.

Von dem lufttrockenen Material wurde durch Herrn Horace L. W ells eine Analyse ausgeführt, welche ergab :

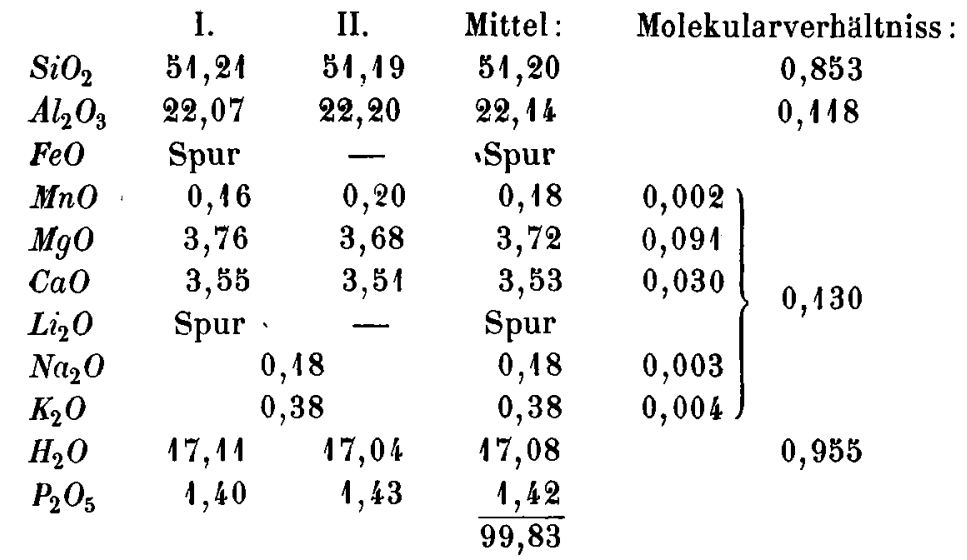

Der Gehalt an Phosphorsäure beweist, dass eine kleine Menge Apatit beigemengt war, und es musste daher die entsprechende Menge $(1,86) \mathrm{CaO}$ in Abzug gebracht werden. Die Analyse stimmt leidlich mit denen des Montmorillonit von Montmorillon in Frankreich uberein - und nähert sich ausserdem derjenigen eines ähnlichen Thonminerals von Macskamezò in Siebenburgen, welches $\mathrm{Hel} \mathrm{mhack}$ er beschrieb*).

Wie bereits erwähnt, war das Mineral unmittelbar nach der Auffindung sehr feucht und plastisch. Es schien nun von einigem Interesse zu bestimmen, in welcher Weise dasselbe sein Wasser verliert. Zwei Gramm des Iufttrockenen Minerals wurden in den Exsiccator uber Schwefelsäure gebracht; wiederholte Wägungen zeigten, dass dasselbe während 6-7 Wochen fortwährend Wasser verlor, im Ganzen 9,80\% ; der gesammte Verlust nach dem Gluhen war ungefähr 17\%.

Die specielle Beziehung dieses Montmorillonit zu den Spodumenpseudomorphosen lässt sich nicht bestimmen. Die Thatsache, dass derselbe so nahe zusammen mit dem Spodumen, wie mit dem Cymatolith vorkommt, scheint dafur zu sprechen, dass er seine Entstehung dem ersteren verdankt, nur musste er dann einem späteren Stadium des Zersetzungsprocesses angehören, da er fast ganz frei von Alkalien ist. Dagegen scheint die Vermutbung, dass er aus dem Feldspath entstanden, und dass hierdurch die Alkalien zur Zersetzung des Spodumen frei geworden seien, auf den ersten Anblick plausibel ; aber erstens ist der Feldspath des Ganges da, wo er mit Heftes.

*) Ts chermak's min. u. petrogr. Mittheil. 1879, 251. (Auszug am Schlusse dieses 
dem Montmorillonit vorkommt, ganz frisch und unzersetzt, und dann ist, soweit unsere Beobachtungen reichen, die Menge des letzteren dafür viel zu gering. Eher dürfte er daher das lokale Resultat einer weitergehenden Zersetzung des Gymatolith darstellen. Eine beslimmte Meinung hieruber abzugeben, fühlen wir uns jedoch zur Zeit ausser Stande.

Am Schlusse dieser Mittheilung müssen wir den Herren Penfield, Wells und Dewey, denen wir für die hier mitgetheilten Analysen verpflichtet sind, unseren Dank aussprechen.

\section{Erklärung der Tafel IV.}

Die Buchstaben in den Figuren bedeuten : $a=$ Albit (wie erwähnt, enthält derselbe. meist Glimmerschuppen und geht dadurch in Cymatolith über), $c=$ Cymatolith, $g=$ Muscovit, $k=$ Killinit, $m=$ Mikroklin, $s=$ Spodumen, $\beta=\beta$-Spodumen.

Fig. 1a-1c. Drei Querschnitte in circa fünf Zoll Abstand aus einem einzigen Krystall von 15 Zoll Länge und 41/2 Zoll Breite. 1a, nahe dem ausgebildeten Ende, besteht wesentlich aus $\beta$-Spodumen $(\beta)$, mit Cymatolith $(c)$ an den Kanten und ein wenig glasigem. Spodumen $(s)$ an der unteren Seite. $4 \mathrm{~b}$ zeigt nur $\beta$-Spodumen und Cymatolith, letzteren in grösserer Menge als 1a. 1 c, vom unteren Ende des Krystalls, besteht nur aus Cymatolith.

Fig. 2. Querschnitt eines $41 / 2$ Zoll breiten, ganz in Cymatolith verwandelten Krystalls ; zu bemerken ist die verworrene und gewundene Structur dieses Minerals und die Richtung seiner Fasern senkrecht zu den Seiten.

Fig. 3. Theilweiser longitudinaler Schnitt; das Innere besteht aus feinkörnigem Albit $(a)$, mit linearen Partien von grobkörnigem spaltbarem Mikroklin $(m)$, das Aeussere aus Cymatolith $(c)$.

Fig. 4. Fragment eines Krystalls; körniger Albit $(a)$ umschliesst Mikroklin $(m)$.

Fig. 5. Querschnitt eines grossen Krystalls, dessen Aussenseite zerbrochen und unregelmässig. Derselbe besteht grösstentheils aus klarem rothen Spodumen $(s)$, durchzogen von Streifen von $\beta$-Spodumen $(\beta)$ parallel den Spaltungsrichtungen; am Rande auch etwas Cymatolith $(c)$.

Fig. 6. Körniger Albit ( $a)$ und Cýmatolith (c); daneben einige Lamellen von Glimmer $(g)$.

Fig. 7. Querschnitt eines grossen Krystalls in natürlicher Grösse ; das Innere besteht. aus faserigem Albit $(a)$, das Aeussere aus Cymatolith $(c)$.

Fig. 8. Spodumen $(s)$ in vereinzelten Partien, umgeben von radial-faserigem Cymatolith $(c)$, daneben etwas $\beta$-Spodumen, körniger Albit $(a)$ und wenige Glimmerlamellen $(g)$.

Fig. 9. Fragment, bestehend aus Killinit $(k)$ mit schmalen Streifen von Cymatolith $(c)$, nahe parallel den ursprünglichen Spaltungsrichtungen des Spodumen.

Fig. 10. Querschnitt eines grossen Krystalls $(71 / 2$ Zoll breit), zusammengesetzt aus Albit $\{a)$ und körnigem Mikroklin $(m)$.

Fig. 11, 13. Spaltungsplatten von Mikroklin in körnigem Albit eingelagert und in jedem der beiden Krystallfragmente parallel orientirt.

Fig. 12. Fragment eines Krystalls mit $\beta$-Spodumen $(\beta)$ in Albit $(a)$ eingeschlossen, der ảussere Theil aus Killinit bestehend.

Fig. 14. Theil eines Krystalls, zusammengesetzt aus Spodumen $(s)$, Cymatolith davon ausstrahlend $(c)$ und körnigem Albit $(a ; \beta$-Spodumen in einem den Spodumen durchsetzenden Streifen. 